\title{
ON VISCOSITY SOLUTIONS TO THE DIRICHLET PROBLEM FOR ELLIPTIC BRANCHES OF INHOMOGENEOUS FULLY NONLINEAR EQUATIONS
}

\author{
Marco Cirant and Kevin R. Payne
}

\begin{abstract}
For scalar fully nonlinear partial differential equations $F\left(x, D^{2} u(x)\right)=0$ with $x \in \Omega \Subset \mathbb{R}^{N}$, we present a general theory for obtaining comparison principles and well posedness for the associated Dirichlet problem, where $F(x, \cdot)$ need not be monotone on all of $\mathcal{S}(N)$, the space of symmetric $N \times N$ matrices. We treat admissible viscosity solutions $u$ of elliptic branches of the equation in the sense of Krylov [20] and extend the program initiated by Harvey and Lawson [11] in the homogeneous case when $F$ does not depend on $x$. In particular, for the set valued map $\Theta$ defining the elliptic branch by way of the differential inclusion $D^{2} u(x) \in \partial \Theta(x)$, we identify a uniform continuity property which ensures the validity of the comparison principle and the applicability of Perron's method for the differential inclusion on suitably convex domains, where the needed boundary convexity is characterized by $\Theta$. Structural conditions on $F$ are then derived which ensure the existence of an elliptic map $\Theta$ with the needed regularity. Concrete applications are given in which standard structural conditions on $F$ may fail and without the request of convexity conditions in the equation. Examples include perturbed Monge-Ampère equations and equations prescribing eigenvalues of the Hessian.
\end{abstract}

2010 Mathematics Subject Classification: 35J60, 35J70, 35D40, 35B51.

Key words: Comparison principles, Perron's method, admissible viscosity solutions, elliptic branches.

\section{Contents}

1. Introduction

2. Admissible viscosity solutions of elliptic branches $\quad 537$

2.1. Elliptic sets and their duals 537

2.2. Elliptic maps and elliptic branches $\quad 539$

2.3. Weak solutions of differential inclusions associated to elliptic maps

3. The comparison principle for uniformly continuous elliptic maps 
3.2. The semi-convex case for arbitrary elliptic maps

3.3. The general case for uniformly continuous elliptic maps

4. Boundary convexity and the Dirichet problem for ellipitc maps

4.1. Elliptic cones and boundary convexity

4.2. Perron's method and the Dirichlet problem

5. Applications to fully nonlinear PDEs

5.1. Structure conditions, the comparison principle, and admissible domains

5.2. Solvability theorems for concrete examples

5.3. The comparison principle for admissible viscosity solutions 572

References

\section{Introduction}

In this work, we will study continuous solutions $u$ of the Dirichlet problem for second order fully nonlinear PDEs of the form

$$
\begin{array}{ll}
F\left(x, D^{2} u(x)\right)=0, & x \in \Omega, \\
u(x)=\varphi(x), & x \in \partial \Omega,
\end{array}
$$

where $\Omega \subset \mathbb{R}^{N}$ is a bounded open domain with $C^{2}$ boundary and $\varphi$ and $F$ are given continuous functions. More precisely, we will examine the validity of the comparison principle for (1.1) and the well posedness of(1.1)-(1.2) by way of Perron's method for admissible viscosity solutions $u$ of elliptic branches of the equation in the sense of Krylov [20]. In particular, we will not assume that $F(x, \cdot)$ is a monotone function on the entire space $\mathcal{S}(N)$ of symmetric $N \times N$ matrices. Moreover, we will seek minimal structural conditions on $F$ in order to operate in Krylov's framework, which shifts attention from the particular form of $F$ to its zero locus as a means of formulating a general notion of ellipticity. Exploiting the freedom of the Krylov framework and the lack of $(u, D u)$ dependence in (1.1), we will obtain comparison principles and well posedness of the Dirichlet problem under structural conditions on $F$ which are in some cases weaker than what would be required in framework of classical viscosity solutions. This classical framework, as presented in Crandall-Ishii-Lions [9], of course applies to more general equations of the form $F\left(x, u(x), D u(x), D^{2} u(x)\right)=0$. That improvements to the classical viscosity theory might be possible in Krylov's setting is suggested by the program initiated by Harvey and Lawson [11] for homogeneous equations $F\left(D^{2} u(x)\right)=0$ and we will generalize their approach to include inhomogeneous equations (1.1). 
Before giving a detailed description of the results obtained and the methods employed, we give a few model equations which illustrate some of the novelty in our applications to the well posedness for (1.1)-(1.2) by Perron's method. In all cases, $f$ is a uniformly continuous real valued function. First, for the perturbed Monge-Ampère equation

$$
\operatorname{det}\left(D^{2} u(x)+M(x)\right)-f(x)=0,
$$

if $f \geq 0$ and if $M$ is a uniformly continuous $\mathcal{S}(N)$-valued function and $\Omega$ is strictly convex, then a unique solution will be found for the branch determined by the constraint $\operatorname{det}\left(D^{2} u(x)+M(x)\right) \geq 0$ in Theorem 5.9. Standard structural conditions may fail for (1.3) in cases that we can treat. For example, the well known condition (3.14) of Crandall-IshiiLions [9] may impose an additional regularity property on $M$ in terms of a lower bound on the order at which coefficients of the matrix are allowed to vanish. We need no such restriction, requiring only that $M$ be uniformly continuous. This is discussed in Remark 5.10 and a simple example is provided. Next, in dimension $N=2$, if $f \geq 0$ and $\Omega$ is strictly convex then the equation

$$
u_{x_{11}} u_{x_{22}}-f(x)=0
$$

admits unique solutions for two distinct branches (see Theorem 5.13) even though along one of the branches, $F(x, A)=A_{11} A_{22}-f(x)$ is not monotone (see Remark 5.14). These two examples illustrate improvements to the classical theory. Finally, for the equation which prescribes the $k$ th-eigenvalue of the Hessian

$$
\lambda_{k}\left(D^{2} u(x)\right)-f(x)=0,
$$

if $\Omega$ is suitably convex a unique solution will be found in Theorem 5.11 where $F_{k}(x, A)=\lambda_{k}(A)-f(x)$ need not be concave (see Remark 5.12), which cuts this example off from other general approaches. See also the discussion at the end of the introduction.

We begin our technical description with some remarks about the general methodology. In the Krylov framework, an elliptic branch of (1.1) is encoded by a set-valued function $\Theta: \Omega \rightarrow \wp(\mathcal{S}(N))$ whose values $\Theta(x)$ must be an elliptic set; that is, a non empty, closed, and proper subset of $\mathcal{S}(N)$ which is stable under sums with non-negative elements in $\mathcal{S}(N)$. The PDE will be replaced by the differential inclusion

$$
D^{2} u(x) \in \partial \Theta(x) \text { for each } x \in \Omega
$$

and $\Theta$ will give an elliptic branch of (1.1) provided that

$$
\partial \Theta(x) \subset \Gamma(x):=\{A \in \mathcal{S}(N): F(x, A)=0\} \text { for each } x \in \Omega,
$$


where obviously one assumes that $\Gamma(x) \neq \emptyset$. A natural definition of viscosity solutions for (1.6) can be given which uses the elliptic map $\Theta$ as an admissibility constraint (see Definition 2.7). In this way, one can temporarily forget the particular form of the PDE and shift focus to the zero locus $\Gamma(x)$ and the elliptic map $\Theta$. One might discover new structural conditions for $F$ by way of properties that the map $\Theta$ must satisfy in order to ensure a comparison principle and to implement Perron's method. Implementing this scheme for a given PDE (1.1) is a two step process: show that an elliptic branch $\Theta$ exists for which the weak solutions of (1.6) are admissible viscosity solutions of (1.1) and determine conditions under which one can handle (1.6).

In the homogeneous case, where the elliptic maps are constant, Harvey and Lawson [11] treated the differential inclusion $D^{2} u(x) \in \partial \Theta$ in an original and elegant way and show that no additional assumptions on $\Theta$ are necessary. The key ingredients are a notion of duality for elliptic sets where the dual set $\widetilde{\Theta}$ is defined in a set theoretic way

$$
\widetilde{\Theta}:=-\left[\Theta^{\circ}\right]^{c}
$$

(where $\Theta^{\circ}$ and $\Theta^{c}$ denote the interior and complement of $\Theta \in \mathcal{S}(N)$ ) and the use of subaffine functions which are upper semicontinuous functions satisfying a comparison principle with respect to affine functions (see Definition 2.9). These ingredients make the proof of the comparison principle particularly transparent and yield a characterization of the boundary convexity needed for Perron's method in terms of $\Theta$ and $\widetilde{\Theta}$. While many interesting and geometrically important examples are discussed in [11], the question of structural conditions on a general $F$ which ensure the existence of elliptic branches is not treated. In the inhomogeneous setting that we treat, where the elliptic maps are not constant, we show that a natural uniform continuity property on $\Theta$ ensures a suitable abstract theory at the level of the differential inclusion (1.6). Moreover, we address the question of suitable structural conditions on $F$ in order to apply the abstract theory to a given PDE (1.1).

We first describe the abstract results concerning the differential inclusion (1.6) which need not bound to any particular PDE (1.1). For any elliptic map $\Theta$, one defines $\Theta$-subharmonic functions as those upper semicontinuous functions that are viscosity subsolutions of (1.6), where one uses $\Theta(x)$ as a pointwise constraint. As in the homogeneous case [11] of constant elliptic maps, the key to the development is a characterization of $\Theta$-subharmonic functions in terms of duality and the space of subaffine functions (whose elements have a natural pointwise characterization). In particular, by using the duality (1.7) pointwise, one has that 
a lower semicontinuous function $u$ is $\Theta$-superharmonic if and only if $-u$ is $\widetilde{\Theta}$-subharmonic. More importantly, in Theorem 2.11 we prove that an upper semicontinuous $u$ function is $\Theta$-subharmonic in $x \in \Omega$ if and only if

$u+v$ is subaffine in $x$

for each $v$ which is $C^{2}$ near $x$ and $\widetilde{\Theta}$-subharmonic in $x$.

By duality, one has a similar characterization of $\Theta$-superharmonic functions $u$. As in the homogeneous case [11], this duality-subaffine reformulation (1.8) shows that the comparison principle for a pair of $\Theta, \widetilde{\Theta}$-subharmonic functions is a consequence of the so-called subaffine theorem, which states that

$$
u+v \text { is subaffine in } \Omega \text { if } u, v \text { are } \Theta, \widetilde{\Theta} \text {-subharmonic in } \Omega \text {. }
$$

The proof of the subaffine theorem (1.9) for upper semicontinuous $u, v$ requires the validity of certain basic properties of $\Theta$-subharmonic functions used in an approximation argument. In the inhomogeneous case we treat, some of these basic properties require the uniform Hausdorff continuity of the elliptic map $\Theta$, which is to say the uniform continuity of $\Theta$ when one places the Hausdorff metric on the collection of closed subsets of $\mathcal{S}(N)$. Proposition 3.3 shows that for an elliptic map $\Theta$, the uniform Hausdorff continuity is equivalent to the following property: for every $\varepsilon>0$ there exists $\delta=\delta(\varepsilon)$ such that

$$
\begin{aligned}
& x, y \in \Omega \text { with }|x-y|<\delta \\
& \quad \Rightarrow \Theta(x)+\varepsilon I \subset \Theta(y) \text { and } \Theta(y)+\varepsilon I \subset \Theta(x) .
\end{aligned}
$$

In addition, the uniform Hausdorff continuity of $\Theta$ passes to the dual map and extends to the boundary while preserving the ellipticity of $\Theta$ (see Proposition 3.5). In Section 3, we will prove the following comparison principle which is essential for the Perron method.

Theorem 1.1. Let $\Theta$ be a uniformly Hausdorff continuous elliptic map on $\Omega$. If $u \in \operatorname{USC}(\bar{\Omega})$ and $w \in \operatorname{LSC}(\bar{\Omega})$ are $\Theta$-subharmonic and $\Theta$-superharmonic respectively in $\Omega$, then

$$
u \leq w \text { on } \partial \Omega \Rightarrow u \leq w \text { in } \Omega .
$$

This comparison principle yields the uniqueness of $\Theta$-harmonic functions having prescribed continuous boundary values. For the existence of such $\Theta$-harmonic functions, as in the homogeneous case [11], the needed boundary convexity of $\Omega$ can be characterized completely in terms of $\Theta$ 
and $\widetilde{\Theta}$ and their associated elliptic cones. An elliptic cone $\vec{\Theta}$ is an elliptic set such that $t A \in \vec{\Theta}$ for each $t \geq 0$ and each $A \in \vec{\Theta}$. Elliptic sets determine an elliptic cone by the formula

$$
\vec{\Theta}:=\overline{\left\{A \in \mathcal{S}(N): \exists t_{0}=t_{0}(A) \in \mathbb{R} \text { such that } t A \in \Theta, \forall t \geq t_{0}\right\}} .
$$

One says that $\partial \Omega$ with a local defining function $\rho \in C^{2}$ near $x \in \partial \Omega$ is strictly $\vec{\Theta}$-convex at $x$ if

$$
\left.D^{2} \rho(x)\right|_{T_{x} \partial \Omega}=\left.B\right|_{T_{x} \partial \Omega} \text { for some } B \in \vec{\Theta}^{\circ},
$$

where $T_{x} \partial \Omega$ is the tangent space at $x$. The uniform Hausdorff continuity of $\Theta$ ensures that the elliptic cone map defined pointwise by (1.12) is constant, as shown in Proposition 4.3. If $\Omega$ is strictly $\vec{\Theta}$-convex, then one can construct a global defining function $\rho$ which is $\vec{\Theta}$-subharmonic on $\bar{\Omega}$ and such that suitable quadratic perturbations of $\rho$ are $\Theta$-subharmonic on $\Omega$ (see Theorem 4.4). The same holds for the dual map $\widetilde{\Theta}$. These auxiliary functions coming from the boundary convexity are used as barriers in the implementation of Perron's method. The following well-posedness result for the Dirichlet problem will be proved in Subsection 4.2.

Theorem 1.2. Let $\Omega \subset \mathbb{R}^{N}$ be a bounded domain with $\partial \Omega$ of class $C^{2}$ and let $\Theta$ be a uniformly Hausdorff continuous elliptic map on $\bar{\Omega}$. Suppose that $\partial \Omega$ is both strictly $\vec{\Theta}$-convex and strictly $\overrightarrow{\widetilde{\Theta}}$-convex. Then for each $\varphi \in C(\partial \Omega)$, there exists a unique $u \in C(\bar{\Omega})$ which is a $\Theta$-harmonic in the sense of Definition 2.7 and such that $u=\varphi$ on $\partial \Omega$.

We will apply this abstract theory to admissible viscosity solutions of elliptic branches of (1.1) in various ways. We first introduce a general situation in which $F$ is increasing along a given background elliptic map $\Phi$. More precisely, we consider $F \in C(\Omega \times \mathcal{S}(N), \mathbb{R})$ for which there is an elliptic map $\Phi$ such that the following conditions hold:

$$
\begin{gathered}
F(x, A) \geq F(x, B), \forall x \in \Omega, \forall A, B \in \Phi(x) \text { such that } A \geq B ; \\
\forall x \in \Omega \text { there exists } A \in \Phi(x) \text { such that } F(x, A)=0 ; \\
\partial \Phi(x) \subset\{A \in \mathcal{S}(N): F(x, A) \leq 0\} \text { for each } x \in \Omega .
\end{gathered}
$$

In Proposition 5.1, we show that an elliptic branch of (1.1) can be defined by

$$
\Theta(x):=\{A \in \Phi(x): F(x, A) \geq 0\}
$$

and we formulate a notion of a $\Phi$-admissible viscosity solution of (1.1) in Definition 5.2. In Proposition 5.3, we show that $u \in C(\Omega)$ is $\Theta$-harmonic 
if and only if $u$ is a $\Phi$-admissible viscosity solution, provided that the following non-degeneracy condition holds

$$
F(x, A)>0 \text { for each } x \in \Omega \text { and each } A \in \Theta(x)^{\circ} .
$$

These results are novel even in the homogeneous case. In this setting, our principal structural condition is: for all small $\varepsilon>0$ there exists $\delta=\delta(\varepsilon)$ such that

(1.19) $F(y, A+\varepsilon I) \geq F(x, A), \forall A \in \Phi(x), \forall x, y \in \Omega$ such that $|x-y|<\delta$.

Proposition 5.4 shows that (1.19) ensures that $\Theta$ defined by (1.17) is uniformly Hausdorff continuous and hence the abstract Theorems 1.1 and 1.2 can be applied. The following result summarizes these considerations and is proven in Subsection 5.1.

Theorem 1.3. Let $\Phi$ be a uniformly Hausdorff continuous elliptic map on $\Omega$ and $F \in C(\Omega \times \mathcal{S}(N), \mathbb{R})$ such that (1.14), (1.15), (1.16), (1.18), and (1.19) hold. Then the elliptic map $\Theta$ defined by (1.17) extends to a uniformly Hausdorff continuous map on $\bar{\Omega}$ and the comparison principle of Theorem 1.1 holds. Moreover, if $\partial \Omega$ is of class $C^{2}$ and strictly $\vec{\Theta}, \vec{\Theta}$-convex, then for each $\varphi \in C(\partial \Omega)$, there exists a unique $u \in C(\bar{\Omega})$ which is a $\Phi$-admissible viscosity solution of (1.1) in the sense of Definition 5.2 and $u=\varphi$ on $\partial \Omega$.

In Proposition 5.5, we describe the interiors of the elliptic cones which are needed for the boundary convexity assumptions on $\Omega$ of Theorem 1.3. Equations which fit into this general situation include (1.3) for which standard structural conditions such as (5.12)-(5.13) may fail, as noted above. In the homogeneous case, this situation means that $F=F(A)$ is increasing along an elliptic set $\Theta$ and (1.19) always holds as do the standard conditions (5.12)-(5.13). This shows that improvements to the classical theory are not seen in the homogeneous case. This situation can be relaxed to allow for $\Phi=\mathcal{S}(N)$ as discussed in Remark 5.8, which then covers examples such as (1.5). The abstract theory also applies to examples such as (1.4) even for the branches on which $F$ is not monotone, and hence cannot be treated in the $\Phi$-admissible setting. Finally, linear equations with $F(x, A)=\operatorname{tr}[a(x) A]-f(x)$ are briefly discussed in Subsection 5.3, where the natural elliptic maps will typically fail to be uniformly Hausdorff continuous. A remedy for this failure is given by truncating where the Hessian is large in order to implement the inherently nonlinear theory presented here.

We conclude this introduction with a few additional remarks and comparisons with the literature. Since we have not assumed that $F(x, A)$ is 
monotone in $A$ on all of $\mathcal{S}(N)$, one is cut off from a large portion of the classical viscosity literature, and this is a major reason for attempting to implement viscosity methods in Krylov's framework of elliptic branches. It should be mentioned that classical viscosity approaches have been employed without monotonicity on all of $\mathcal{S}(N)$; however, they are typically ad hoc approaches which exploit the particular structure of special but important classes of equations, beginning with those of Monge-Ampère type as treated in Section V.3 of Ishii-Lions [15] or for prescribed curvature equations as treated in the paper of Trudinger [23]. We have aimed at a general theory which also encompasses the case with global monotonicity and examples with no monotonicity at all.

We consider equations $F\left(x, D^{2} u\right)=0$ without explicit dependence on $u$ and its gradient $D u$. This has several consequences. As already noted, the proof of the comparison principle reduces to the subaffine theorem, where the maximum principle for subaffine functions takes the place of having to study the maximum principle for semicontinuous functions as needed in the classical scheme of doubling variables and then penalizing, as introduced in Ishii [14] (see also Crandall-Ishii [8]). Such an approach, while working well in a more general context results in structural conditions such those recalled in Remark 5.10 which we do not require here. In addition, since we have no explicit $u$ dependence in (1.1), we can never have strict monotonicity in $u$, which is known to temper the demands on ellipticity for the validity of the comparison principle. A formulation of the ellipticity which is sufficient is given in (1.19), which we refer to as being non-totally degenerate, do to its similarity with the condition of Bardi-Mannucci [2] for equations which can also depend on $(u, D u)$ (see Remark 5.7). Other recent attempts to address the comparison principle in the absence of strict monotonicity in $u$ include Barles-Busca [3], Kawohl-Kutev [17], and Luo-Eberhard [21], where again monotonicity on all of $\mathcal{S}(N)$ is used. Moreover, ignoring that difference for a moment, in $[3]$ there is no $x$ dependence and the structural condition (1.6) of [17] cannot be satisfied for equations independent of $(u, D u)$. The structural conditions of [21] see more similar to ours, but the presence of $(u, D u)$ again plays a role.

There is of course also an extensive literature for treating fully nonlinear equations of the form (1.1) by means other than viscosity techniques. This is particularly true for special classes of equations such as those of Monge-Ampère type or for $F(x, A)=G(A)-f(x)$ with $G$ a symmetric function of $\lambda_{k}(A)$. For example, maximum principles and the continuity method are employed in Section 8 of Caffarelli-Nirenberg-Spruck $[\mathbf{6}, \mathbf{7}]$ to treat smooth solutions of such equations including (1.3) with $f>0$. 
There is also an extensive literature on equations which can be put into Bellman or Bellman-Isaacs form. In particular, Krylov [18] uses this approach to treat strong solutions of (1.3) on balls if $f \geq 0$ and $M$ are $C^{2}$ (see Example 8.2.4 and the comments in Section 8.9 of $[\mathbf{1 8}]$ ). Moreover, the solutions can be shown to be $C^{3, \alpha}$ up to the boundary if $f>0$. Solutions should be semi-convex, lying on the elliptic branch $D^{2} u+M \geq 0$ which is the branch we also consider. Finally, one should consider Krylov's pioneering paper [20] (see also the related [19]) on elliptic branches in which well-posedness results for the Dirichlet problem for equations involving elementary symmetric polynomials in the eigenvalues of $D^{2} u$ are shown. An important point is that when the elliptic sets $\Theta(x)$ (or their complements) are convex, then canonical forms of the elliptic branch can be converted into Bellman form, to which solvability results for general nonlinear PDE by barrier techniques apply. We make no assumption on the convexity of $\Theta(x)$. Examples such as (1.5) treated Theorem 5.11 have branches which are not convex.

\section{Admissible viscosity solutions of elliptic branches}

In this section, we will present the mathematical formalism for treating viscosity solutions of a fully nonlinear PDE of the form $F\left(x, D^{2} u\right)=0$ in which $F(x, A)$ may not be monotone in $A$ over the entire space of symmetric matrices. We will exploit Krylov's general notion of ellipticity [20] and show that the natural definition of admissibile viscosity solutions of the differential inclusion describing the branch can be reformulated in terms of Harvey-Lawson duality and subaffine functions.

We first fix a few notations. In all that follows, $\mathcal{S}(N)$ denotes the space of symmetric $N \times N$ matrices, which carries the usual partial ordering of the associated quadratic forms. We will denote by $\lambda_{1}(A) \leq \cdots \leq \lambda_{N}(A)$ the eigenvalues of $A \in \mathcal{S}(N)$ and we will denote by

$$
\mathcal{P}:=\{P \in \mathcal{S}(N): P \geq 0\}=\left\{P \in \mathcal{S}(N): \lambda_{1}(P) \geq 0\right\}
$$

the set of non-negative symmetric matrices. We will denote by $\wp(\mathcal{S}(N))$ := $\{\Phi: \Phi \subset \mathcal{S}(N)\}$ and use the notations $\bar{\Phi}, \Phi^{\circ}$, and $\Phi^{c}$ for the closure, interior and complement of $\Phi \in \wp(\mathcal{S}(N))$. Finally, we make use of upper and lower semicontinuous functions:

$$
\begin{aligned}
& \operatorname{USC}(\Omega)=\left\{u: \Omega \rightarrow[-\infty, \infty): u\left(x_{0}\right) \geq \limsup _{x \rightarrow x_{0}} u(x), \forall x_{0} \in \Omega\right\} ; \\
& \operatorname{LSC}(\Omega)=\left\{u: \Omega \rightarrow(-\infty, \infty]: u\left(x_{0}\right) \leq \liminf _{x \rightarrow x_{0}} u(x), \forall x_{0} \in \Omega\right\} .
\end{aligned}
$$

2.1. Elliptic sets and their duals. We begin with a brief review of the notion of elliptic sets and their duals, which provide the framework for the discussion. 
Definition 2.1. A subset $\Theta$ of $\mathcal{S}(N)$ is called an elliptic set if $\Theta$ is closed, non empty, proper and satisfies the positivity property

$$
\Theta+\mathcal{P} \subset \Theta
$$

that is, $A+P \in \Theta$ for each $A \in \Theta$ and each $P \in \mathcal{P}$, where $\mathcal{P}$ is defined by (2.1). The collection of all elliptic subsets will be denoted by $\mathcal{E}$.

In the original definition of elliptic sets, Krylov $[\mathbf{2 0}]$ took $\Theta$ to be an open subset, but we have followed [11] by taking $\Theta$ to be closed. This will be useful since the collection of closed subsets $\mathcal{K}(\mathcal{S}(N))$ of $\mathcal{S}(N)$ forms a metric space with respect to the Hausdorff distance, which will be used to describe the regularity of $\mathcal{E}$-valued maps.

As shown in Section 3 of $[\mathbf{1 1}], \Theta$ has non empty interior since each $A \in \Theta$ can be written as the limit as $\varepsilon \rightarrow 0^{+}$of $A+\varepsilon I \in \Theta+\mathcal{P}^{\circ}$ and one has

$$
\Theta=\overline{\Theta^{\circ}} .
$$

In addition, an elliptic set $\Theta$ is completely determined by its boundary in the sense that

$$
\Theta=\{B+t I: B \in \partial \Theta, t \geq 0\},
$$

as noted in Remark 2.5 of [20].

Duality is defined in a set theoretic way.

Definition 2.2. For each $\Theta \in \wp(\mathcal{S}(N))$, define the dual set $\widetilde{\Theta} \in \wp(\mathcal{S}(N))$ by

$$
\widetilde{\Theta}=\left[-\Theta^{\circ}\right]^{c}=-\left[\Theta^{\circ}\right]^{c} .
$$

An important example is given by the dual to the elliptic set $\mathcal{P}$, where

$$
\widetilde{\mathcal{P}}=\left\{A \in \mathcal{S}(N): \lambda_{N}(A) \geq 0\right\}
$$

is also an elliptic set. Duality preserves the ellipticity of $\Theta$ and is well behaved with respect to various operations.

Proposition 2.3. For arbitrary subsets $\Theta, \Theta^{\prime}$ of $\mathcal{S}(N)$, one has the following properties:

(a) $\widetilde{\Theta}$ is elliptic if and only if $\Theta$ is elliptic;

(b) the dual of $\widetilde{\Theta}$ is $\Theta$;

(c) if $\Theta \subset \Theta^{\prime}$ then $\widetilde{\Theta^{\prime}} \subset \widetilde{\Theta}$;

(d) $\widetilde{\Theta+A}=\widetilde{\Theta}-A$ for every $A \in \mathcal{S}(N)$.

These formulas and others are shown in Section 3 of [11]. Duality combined with ellipticity yields the following important facts. 
Proposition 2.4. For elliptic sets $\Theta$, one has the following properties:

(a) $\partial \Theta=\Theta \cap(-\widetilde{\Theta})=\Theta \backslash \Theta^{\circ}$;

(b) $A \in \Theta$ if and only if $A+\widetilde{\Theta} \subset \widetilde{P}$.

Property (a) follows directly from the definition and requires only that $\Theta$ be closed and is not trivial if $\Theta$ is proper. Property (b) is proven in Lemma 4.3 of $[\mathbf{1 1}]$ and yields the following alternate characterization of the dual for elliptic sets

$$
\widetilde{\Theta}=\{B \in \mathcal{S}(N): B+\Theta \subset \widetilde{P}\} .
$$

2.2. Elliptic maps and elliptic branches. Next we briefly review Krylov's notion of elliptic branches for a fully nonlinear PDE of the form (1.1). Elliptic branches will be encoded by elliptic maps, which are nothing other than elliptic set valued maps.

Definition 2.5. A set valued map $\Theta: \Omega \rightarrow \wp(\mathcal{S}(N))$ is called an elliptic map if $\Theta$ takes values in the elliptic subsets $\mathcal{E}$ of $\mathcal{S}(N)$; that is, for each $x \in \Omega$ one has

$$
\Theta(x) \varsubsetneqq \mathcal{S}(N), \Theta(x) \text { is closed and nonempty, } \Theta(x)+\mathcal{P} \subset \Theta(x) .
$$

Since $\Theta$ is just an $\mathcal{E}$-valued map, all of the properties listed in Subsection 2.2 hold pointwise; that is, for each $\Theta(x)$ with $x \in \Omega$. In particular, one has a natural dual map $\Theta$ with $\widetilde{\Theta}(x):=\widetilde{\Theta(x)}$ for each $x \in \Omega$. Moreover, each fixed elliptic set $\Theta$ can be identified as a constant elliptic map so that the homogeneous case treated in [11] is strictly included in the present theory.

We now turn to the notion of an elliptic branch. Consider the fully nonlinear PDE of the form (1.1); that is,

$$
F\left(x, D^{2} u(x)\right)=0, \quad x \in \Omega,
$$

with $\Omega \Subset \mathbb{R}^{N}$ and $F: \Omega \times \mathcal{S}(N) \rightarrow \mathbb{R}$ continuous such that the zero locus

$$
\Gamma(x):=\{A \in \mathcal{S}(N): F(x, A)=0\} \text { with } x \in \Omega
$$

is closed and assumed to be non empty for each $x \in \Omega$.

Definition 2.6. An elliptic branch of (2.9) is the differential inclusion

$$
D^{2} u(x) \in \partial \Theta(x) \text { for each } x \in \Omega,
$$

provided that $\Theta$ is an elliptic map and the following branch condition holds:

$$
\partial \Theta(x) \subset \Gamma(x) \text { for each } x \in \Omega,
$$

where $\Gamma(x)$ is the zero locus $(2.10)$. 
Following Krylov, one could say that the PDE (2.9) is elliptic if it admits an elliptic branch $\Theta$, which need not be unique. Moreover, $\Theta$ depends only on the zero locus in (2.10) and not on the particular form of $F$. A given $F$ is said to be a canonical form for $\Theta$ if for each $x \in \Omega$ one has:

$$
\begin{cases}F(x, A)>0 & \text { for each } A \in \Theta(x)^{\circ}, \\ F(x, A)=0 & \text { for each } A \in \partial \Theta(x), \\ F(x, A)<0 & \text { for each } A \in \Theta(x)^{c} .\end{cases}
$$

When $F$ satisfies (2.13), then one has also

$$
\begin{aligned}
& F(x, A) \text { is concave (convex) in } A \in \mathcal{S}(N) \\
& \Rightarrow \Theta(x)\left(\left[\Theta(x)^{\circ}\right]^{c}\right) \text { is a convex set. }
\end{aligned}
$$

Notice that Proposition 2.4(a) yields

$$
\partial \Theta(x)=\Theta(x) \cap(-\widetilde{\Theta(x)})=\Theta(x) \backslash \Theta(x)^{\circ} .
$$

Hence if $F$ is a canonical form for $\Theta$ and if $u$ is twice differentiable on $\Omega$, then solutions of (2.11) are solutions of (2.9). Moreover classical subsolutions of (2.9) are given by

$$
D^{2} u(x) \in \Theta(x) \text { for each } x \in \Omega,
$$

while classical supersolutions of (2.9) are given by

$$
D^{2} u(x) \notin \Theta(x)^{\circ} \text { for each } x \in \Omega .
$$

Notice that $(2.16)$ is equivalent to $D^{2}(-u)(x) \in \widetilde{\Theta}(x)$ and hence duality allows one to reformulate classical supersolutions as subsolutions to a dual equation. Moreover, for an arbitrary elliptic map $\Theta$ which need not be bound to any particular PDE (2.9), classical solutions to $(2.15) /(2.16)$ will be called $\Theta$-subharmonic/superharmonic in $\Omega$. For $u$ merely upper/lower semicontinuous on $\Omega$, one defines solutions to $(2.15),(2.16)$ and hence (2.11) in a viscosity sense with the elliptic map $\Theta$ playing the role of an admissibility constraint.

\subsection{Weak solutions of differential inclusions associated to ellip-} tic maps. As indicated in the previous section, for any elliptic map $\Theta$, weak (sub/super) solutions $u$ to the differential inclusion (2.11) will be defined in the viscosity sense where one can make use of a suitable notion of (reduced) second order differentials. More precisely, for each $x_{0} \in \Omega$ 
define the (reduced) second order superdifferential

$$
\begin{aligned}
J^{+} u\left(x_{0}\right):=\left\{D^{2} \varphi\left(x_{0}\right)\right. & : \varphi \text { is } C^{2} \text { near } x_{0} \\
& \text { and } \left.u-\varphi \text { has a local maximum in } x_{0}\right\}
\end{aligned}
$$

and the (reduced) second order subdifferential

$$
\begin{aligned}
J^{-} u\left(x_{0}\right):=\left\{D^{2} \varphi\left(x_{0}\right): \varphi\right. & \text { is } C^{2} \text { near } x_{0} \\
& \text { and } \left.u-\varphi \text { has a local minimum in } x_{0}\right\} .
\end{aligned}
$$

Other equivalent formulations could be given. For example, it is clear that $\varphi \in \mathcal{C}^{2}$ could be replaced by $\varphi$ a quadratic polynomial satisfying $\varphi\left(x_{0}\right)=u\left(x_{0}\right)$.

Definition 2.7. Let $\Theta$ be an elliptic map on $\Omega$.

(a) A function $u \in \operatorname{USC}(\Omega)$ will be called $\Theta$-subharmonic in $x_{0}$ if

$$
J^{+} u\left(x_{0}\right) \subset \Theta\left(x_{0}\right),
$$

and is said to be $\Theta$-subharmonic in $\Omega$ if (2.19) holds for each $x_{0} \in$ $\Omega$. The spaces of such functions will be denoted by $\Theta \mathrm{SH}\left(x_{0}\right)$ and $\Theta \mathrm{SH}(\Omega)$ respectively.

(b) A function $u \in \operatorname{LSC}(\Omega)$ will be called $\Theta$-superharmonic in $x_{0}$ if

$$
J^{-} u\left(x_{0}\right) \subset\left[\Theta\left(x_{0}\right)^{\circ}\right]^{c} .
$$

(c) A function $u \in C(\Omega)$ will be called $\Theta$-harmonic in $\Omega$ if is both $\Theta$-subharmonic and $\Theta$-superharmonic in $\Omega$.

When the elliptic map $\Theta$ defines and elliptic branch for the PDE (2.9), one defines an admissible viscosity solution of the branch of (2.9) determined by $\Theta$ as a function $u \in C(\Omega)$ which is $\Theta$-harmonic on $\Omega$. Applications to admissible viscosity solutions of PDEs will be given Section 5 . We will provide various ways to make use of the abstract theory for $\Theta$-harmonic functions.

We record a few basic facts concerning Definition 2.7. Since $\left[\Theta\left(x_{0}\right)^{\circ}\right]^{c}=$ $-\widetilde{\Theta}\left(x_{0}\right)$ and $J^{+}(-u)\left(x_{0}\right)=-J^{-} u\left(x_{0}\right)$, one has

(2.21) $u \in \operatorname{LSC}(\Omega)$ is $\Theta$-superharmonic in $x_{0}$

$$
\text { if and only if }-u \in \widetilde{\Theta} \mathrm{SH}\left(x_{0}\right) \text {; }
$$

that is, $\Theta$-superharmonicity can be expressed in terms of subharmonicity for the dual map, as noted earlier for classical solutions. Important 
examples include the constant elliptic maps $\mathcal{P}, \widetilde{\mathcal{P}}$, and $\mathcal{H}=\{A \in \mathcal{S}(N)$ : $\operatorname{tr}(A) \geq 0\}$, where $\widetilde{\mathcal{H}}=\mathcal{H}$. One has:

$$
u \in \widetilde{\mathcal{P}} \mathrm{SH}(\Omega) \Leftrightarrow u \text { is subaffine on } \Omega ;
$$

$$
u \in \mathcal{P S H}(\Omega) \Leftrightarrow u \text { is convex on } \Omega \text { or } u \equiv-\infty \text {; }
$$

$u \in \mathcal{H} \mathrm{SH}(\Omega) \Leftrightarrow u\left(x_{0}\right) \leq \frac{1}{\left|B_{r}\left(x_{0}\right)\right|} \int_{B_{r}\left(x_{0}\right)} u(x) d x$ for each $B_{r}\left(x_{0}\right) \Subset \Omega$.

The equivalence (2.24) is classical and see Proposition 4.5 of [11] for (2.22)-(2.23).

In addition, one has the following coherence property which will be used frequently.

Proposition 2.8. Let $u \in \operatorname{USC}(\Omega)$ be twice differentiable ${ }^{1}$ in $x_{0} \in \Omega$. Then

$$
u \in \Theta \operatorname{SH}\left(x_{0}\right) \Leftrightarrow D^{2} u\left(x_{0}\right) \in \Theta\left(x_{0}\right) .
$$

The forward implication makes use of the Taylor expansion for $u$ and the fact (2.3), while the reverse implication uses only the positivity property $(2.2)$.

We conclude this section with a useful characterization of $\Theta$-harmonicity for elliptic maps, which we will use throughout and which generalizes the characterization of $[\mathbf{1 1}]$ for constant elliptic maps. We begin with the notion of a subaffine function.

Definition 2.9. A function $w \in \operatorname{USC}(\Omega)$ is said to be subaffine in $\Omega$ if for every compact $K \subset \Omega$ and for every affine function $a$

$$
w \leq a \text { on } \partial K \Rightarrow w \leq a \text { on } K \text {. }
$$

Denote by $\operatorname{SA}(\Omega)$ the space of such functions.

One has the following pointwise characterization of subaffine functions, as proved in Lemma 2.2 of [11].

Lemma 2.10. If $w \in \operatorname{USC}(\Omega)$, then $w \in \operatorname{SA}(\Omega)$ if and only if for each $x_{0} \in \Omega$ one has

$\nexists$ a triple $(\varepsilon, r, a)$ with $\varepsilon, r>0$ and an affine function a such that

$$
(w-a)\left(x_{0}\right)=0 \text { and }(w-a)(x) \leq-\varepsilon\left|x-x_{0}\right|^{2}, \forall x_{0} \in B_{r}\left(x_{0}\right) .
$$

We will denote by $\operatorname{SA}\left(x_{0}\right):=\{w \in \operatorname{USC}(\Omega)$ such that (2.26) holds $\}$.

${ }^{1} u(x)=u\left(x_{0}\right)+\left\langle p, x-x_{0}\right\rangle+\frac{1}{2}\left\langle A\left(x-x_{0}\right), x-x_{0}\right\rangle+o\left(\left|x-x_{0}\right|^{2}\right)$ as $x \rightarrow x_{0}$ for some $(p, A) \in \mathbb{R}^{N} \times \mathcal{S}(N)$. Hence $u$ is differentiable in $x_{0}$ with $p=D u\left(x_{0}\right)$ and we denote by $D^{2} u\left(x_{0}\right)$ the matrix $A$. 
Theorem 2.11. Let $\Theta$ be an elliptic map on $\Omega$.

(a) A function $u \in \operatorname{USC}(\Omega)$ is $\Theta$-subharmonic in $x_{0} \in \Omega$ if and only if (2.27) $u+v \in \mathrm{SA}\left(x_{0}\right)$ for all $v \in C^{2}(\Omega)$ such that $D^{2} v\left(x_{0}\right) \in \widetilde{\Theta}\left(x_{0}\right)$.

(b) A function $u \in \operatorname{LSC}(\Omega)$ is $\Theta$-superharmonic in $x_{0} \in \Omega$ if and only if

(2.28) $-u+v \in \mathrm{SA}\left(x_{0}\right)$ for all $v \in C^{2}(\Omega)$ such that $D^{2} v\left(x_{0}\right) \in \Theta\left(x_{0}\right)$.

Proof: Since (b) is equivalent to the statement that $-u \in \widetilde{\Theta} \mathrm{SH}\left(x_{0}\right)$, claim (b) follows from claim (a) by duality as the dual of $\widetilde{\Theta}\left(x_{0}\right)$ is $\Theta\left(x_{0}\right)$. For the claim (a), we argue by contradiction. First, assume that $u \in$ $\Theta \mathrm{SH}\left(x_{0}\right)$ but that (2.27) fails. By Definition 2.7 and Lemma 2.10, there exist $v \in C^{2}(\Omega)$ with $D^{2} v\left(x_{0}\right) \in \widetilde{\Theta}\left(x_{0}\right)$ and a triple $(\varepsilon, r, a)$ such that $(2.29)(u+v-a)\left(x_{0}\right)=0$ and $(u+v-a)(x) \leq-\varepsilon\left|x-x_{0}\right|^{2}, \forall x \in B_{r}\left(x_{0}\right)$.

By reducing $\varepsilon$, we can assume that $v$ satisfies the stronger condition

$$
D^{2} v\left(x_{0}\right) \in \widetilde{\Theta}^{\circ}\left(x_{0}\right)=-\left[\Theta\left(x_{0}\right)^{c}\right] .
$$

Indeed, the perturbation $w:=v+\varepsilon Q_{x_{0}}$ with

$$
Q_{x_{0}}(x)=\frac{1}{2}\left|x-x_{0}\right|^{2}
$$

satisfies $D^{2} w\left(x_{0}\right)=D^{2} v\left(x_{0}\right)+\varepsilon I \in \widetilde{\Theta}^{\circ}\left(x_{0}\right)$ and

$$
(u+w-a)\left(x_{0}\right)=0 \text { and }(u+w-a)(x) \leq-\frac{\varepsilon}{2}\left|x-x_{0}\right|^{2}, \forall x \in B_{r}\left(x_{0}\right) .
$$

For $\varphi:=-v+a$ with $v$ satisfying (2.29) and (2.30), the function $u-\varphi$ has a local maximum in $x_{0}$ and hence $D^{2} \varphi\left(x_{0}\right) \in \Theta\left(x_{0}\right)$ by the hypothesis $u \in \Theta \mathrm{SH}\left(x_{0}\right)$. This gives a contradiction since

$$
D^{2} \varphi\left(x_{0}\right)=-D^{2} v\left(x_{0}\right) \in\left[\Theta\left(x_{0}\right)^{c}\right] .
$$

Conversely, if $u$ satisfies (2.27) but is not $\Theta$-subharmonic in $x_{0}$ then there must exist $\varphi$ which is $C^{2}$ near $x_{0}$ such that $u-\varphi$ has a local maximum in $x_{0}$ but $D^{2} \varphi\left(x_{0}\right) \notin \Theta\left(x_{0}\right)$. One can assume that $\varphi\left(x_{0}\right)=$ $u\left(x_{0}\right)$ and hence there exists $r>0$ such that

$$
(u-\varphi)(x) \leq(u-\varphi)\left(x_{0}\right)=0, \forall x \in B_{r}\left(x_{0}\right) .
$$

For each $\varepsilon>0$ and with $Q_{0}$ defined by (2.31) one has $\left(u-\varphi-\varepsilon Q_{x_{0}}\right)\left(x_{0}\right)=0$ and $\left(u-\varphi-\varepsilon Q_{x_{0}}\right)(x) \leq-\frac{\varepsilon}{2}\left|x-x_{0}\right|^{2}, \forall x \in B_{r}\left(x_{0}\right)$, which by (2.26) says that

$$
u+v_{\varepsilon} \notin \mathrm{SA}\left(x_{0}\right) \text { for each } \varepsilon>0 \text {, where } v_{\varepsilon}:=-\varphi-\varepsilon Q_{x_{0}} .
$$


Since $u$ satisfies $(2.27)$, one concludes that $D^{2} v_{\varepsilon} \notin \widetilde{\Theta}\left(x_{0}\right)$ for each $\varepsilon>0$; that is,

$$
D^{2} \varphi\left(x_{0}\right)+\varepsilon I \in-\left[\widetilde{\Theta}\left(x_{0}\right)\right]^{c}=\left[\Theta\left(x_{0}\right)\right]^{\circ} \text { for each } \varepsilon>0 .
$$

Taking the limit as $\varepsilon \rightarrow 0$ yields $D^{2} \varphi\left(x_{0}\right) \in \Theta\left(x_{0}\right)$, which contradicts the choice of $\varphi$.

\section{The comparison principle for uniformly continuous elliptic maps}

In this section we will prove the comparison principle for uniformly continuous maps, which was stated in Theorem 1.1. As in the homogeneous case of constant elliptic maps [11], duality and the comparison principle for subaffine functions reduce the comparison principle to the subaffine theorem (1.9) for pairs $u, v$ of $\Theta, \widetilde{\Theta}$-subharmonic functions. For any elliptic map $\Theta$, the subaffine theorem holds if $u, v$ are also semiconvex functions. The proof of the subaffine theorem for $u, v$ semicontinuous is accomplished by approximating $u, v$ by sequences of subharmonic and semi-convex functions and then passing to the limit. This will require certain basic properties of $\Theta$-subharmonic functions. Some of these basic properties require regularity of the elliptic map $\Theta$, which come for free in the homogeneous case where $\Theta$ is constant.

We begin with the reduction of the comparison principle to the subaffine theorem.

Proposition 3.1. Let $\Theta$ be any elliptic map on $\Omega$ and let $u \in \operatorname{USC}(\bar{\Omega})$ and $w \in \operatorname{LSC}(\bar{\Omega})$ be $\Theta$-subharmonic and $\Theta$-superharmonic respectively in $\Omega$. Then

$$
u \leq w \text { on } \partial \Omega \Rightarrow u \leq w \text { in } \Omega,
$$

provided that for each $u, v \in \operatorname{USC}(\bar{\Omega})$ one has

$$
u \in \Theta \mathrm{SH}(\Omega), v \in \widetilde{\Theta} \mathrm{SH}(\Omega) \Rightarrow u+v \in \mathrm{SA}(\Omega) .
$$

Proof: Indeed, by the comparison principle (2.25) which defines $u+v \in$ $\mathrm{SA}(\Omega)$, for each $a$ affine one has

$$
u+v \leq a \text { on } \partial \Omega \Rightarrow u+v \leq a \text { in } \Omega,
$$

which is just (3.1) for $a=0$ and $w:=-v \in \operatorname{LSC}(\bar{\Omega})$ and $\Theta$-superharmonic.

A few important observations concerning the validity of the subaffine theorem are in order. First, notice that if (3.2) holds, then the duality 
formula (2.7) for the dual $\widetilde{\Theta}$ of an elliptic set $\Theta$ extends to $\widetilde{\Theta} \mathrm{SH}(\Omega)$ and $\Theta \mathrm{SH}(\Omega)$ in the sense that

$$
\widetilde{\Theta} \mathrm{SH}(\Omega)=\{v \in \operatorname{USC}(\Omega): u+v \in \mathrm{SA}(\Omega) \text { for all } u \in \Theta \operatorname{SH}(\Omega)\} \text {. }
$$

We will show that (3.2) holds provided that the elliptic map $\Theta$ is uniformly Hausdorff continuous and hence we will obtain the duality formula (3.3) for such maps $\Theta$.

Next, notice that the subaffine theorem (3.2) certainly holds if one of the functions $u, v$ are regular. Indeed, if $v \in C^{2}(\Omega)$, then the coherence property Proposition 2.8 yields $D^{2} v(x) \in \widetilde{\Theta}(x)$ for every $x \in \Omega$ and hence $u+v \in \mathrm{SA}(\Omega)$ by the characterization (2.27) of $u \in \Theta \mathrm{SH}(\Omega)$. Since $\widetilde{\widetilde{\Theta}}(x)=\Theta(x)$, the same argument works if $u \in C^{2}(\Omega)$.

Hence, the comparison principle (3.1) holds if one of the functions $u, w$ are $C^{2}$ in a neighborhood of each point $x \in \Omega$. The challenge then is to prove that (3.2) holds if both $u \in \Theta \operatorname{SH}(\Omega)$ and $v \in \widetilde{\Theta} \operatorname{SH}(\Omega)$ are merely semi-continuous.

3.1. Uniform continuity of elliptic maps. As previously noted, the proof of the subaffine theorem for semicontinuous $u, v$ involves approximating them by semi-convex functions which remain $\Theta, \widetilde{\Theta}$ subharmonic (so that Proposition 3.9 below applies along the sequence) and then passing to the limit. This can be done provided that the elliptic map $\Theta$ is uniformly Hausdorff continuous. In this section, we discuss this regularity property for elliptic maps and present the basic properties of $\Theta$-subharmonic functions needed in the approximation argument.

Given $\Phi \subset \mathcal{S}(N)$, we will denote by

$$
N_{\varepsilon} \Phi=\{B \in \mathcal{S}(N):\|B-A\|<\varepsilon \text { for some } A \in \Phi\}=\bigcup_{A \in \Phi} B_{\varepsilon}(A),
$$

the $\varepsilon$-enlargement of the subset $\Phi$ where $\|A\|:=\max _{1 \leq i \leq N}\left|\lambda_{i}(A)\right|$ gives a norm on $\mathcal{S}(N)$. The collection $\mathcal{K}(\mathcal{S}(N))$ of the closed subsets of $\mathcal{S}(N)$ can be equipped with the Hausdorff distance on defined by

$$
d_{\mathcal{H}}(\Phi, \Psi):=\inf \left\{\varepsilon>0: \Phi \subset N_{\varepsilon}(\Psi) \text { and } \Psi \subset N_{\varepsilon}(\Phi)\right\} .
$$

Since $\mathcal{S}(N)$ is complete with respect to the metric $\operatorname{dist}(A, B)=\|A-B\|$, one knows that $\left(\mathcal{K}(\mathcal{S}(N)), d_{\mathcal{H}}\right)$ is a complete metric space (see Proposition 7.3.3 and Proposition 7.3.7 of Burago, Burago, and Ivanov [4], for example). Since the subsets of $\mathcal{S}(N)$ need not be bounded, the metric can take on the value $+\infty$; in particular, one has

$$
d_{\mathcal{H}}(\Phi, \emptyset)=+\infty \text { for each non empty } \Phi \in \mathcal{K}(\mathcal{S}(N)) .
$$


Recalling that elliptic maps take values in the elliptic subsets $\mathcal{E} \subset$ $\mathcal{K}(\mathcal{S}(N))$, a natural notion of uniform continuity is available.

Definition 3.2. An arbitrary map $\Theta: \Omega \rightarrow \mathcal{K}(\mathcal{S}(N))$ will be called uniformly Hausdorff continuous if for each $\eta>0$ there exists $\delta=\delta(\eta)>$ 0 such that

$$
d_{\mathcal{H}}(\Theta(x), \Theta(y))<\eta \text { for each } x, y \in \Omega \text { such that }|x-y|<\delta .
$$

For elliptic maps, the uniform Hausdorff continuity has useful equivalent formulations.

Proposition 3.3. Let $\Theta$ be an elliptic map. Then the following are equivalent:

(a) $\Theta$ is uniformly Hausdorff continuous in the sense of Definition 3.2.

(b) For each $\eta>0$ there exists $\delta=\delta(\eta)>0$ such that

$$
\Theta\left(B_{\delta}(x)\right) \subset N_{\eta}(\Theta(x)) \text { for each } x \in \Omega \text {. }
$$

(c) For each $\eta>0$ there exists $\delta=\delta(\eta)>0$ such that for each $x, y \in \Omega$

$$
|x-y|<\delta \Rightarrow \Theta(x)+\eta I \subset \Theta(y) \text { and } \Theta(y)+\eta I \subset \Theta(x) .
$$

Proof: We will show that $(\mathrm{a}) \Rightarrow(\mathrm{b}) \Rightarrow(\mathrm{c}) \Rightarrow(\mathrm{a})$. First, let $\delta=\delta(\eta / 2)$ be as in the definition of uniform Hausdorff continuity (3.6). It follows that

and hence one has $(3.7)$.

$$
\Theta(y) \subset N_{\eta}(\Theta(x)) \text { for each } y \in B_{\delta}(x),
$$

Next, assume that (3.7) holds. For each $x, y \in \Omega$ with $|x-y|<\delta$ one has

$$
\Theta(x) \subset N_{\eta}(\Theta(y)) \text { and } \Theta(y) \subset N_{\eta}(\Theta(x)) .
$$

For each $A \in \Theta(x)$, the first inclusion in (3.9) yields $A=B+M$ with $B \in \Theta(y)$ and $\|M\|<\eta$ so that

$$
A+\eta I=B+M+\eta I \in \Theta(y)+(M+\eta I) \subset \Theta(y)
$$

since $M+\eta I \in \mathcal{P}$ and $\Theta(y)$ is an elliptic set. Thus the first inclusion in (3.8) holds. A similar argument gives the second inclusion in (3.8).

Finally, let $\delta=\delta(\eta / 2)$ be as in the formulation (3.8) and consider those $x, y \in \Omega$ be such that $|x-y|<\delta$. From the first inclusion in (3.8), given $A \in \Theta(x)$ there exists $B \in \Theta(y)$ such that $A+\frac{\eta}{2} I=B$ and hence

$$
\inf _{B \in \Theta(y)}\|A-B\|<\eta \text { for each } A \in \Theta(x) \text { and each } y \in B_{\delta}(x) \cap \Omega,
$$

which yields

$$
\sup _{A \in \Theta(x)} \inf _{B \in \Theta(y)}|| A-B \|<\eta \text { for each } x, y \in \Omega \text { such that }|x-y|<\delta .
$$


From the second inclusion in (3.8), the same argument yields

$$
\sup _{B \in \Theta(y)} \inf _{A \in \Theta(x)}|| A-B \|<\eta \text { for each } x, y \in \Omega \text { such that }|x-y|<\delta .
$$

Combining (3.10) and (3.11) yields

$$
\max \left\{\sup _{A \in \Theta(x)} \inf _{B \in \Theta(y)}\|A-B\|, \sup _{B \in \Theta(y)} \inf _{A \in \Theta(x)}\|A-B\|\right\}<\eta,
$$

but the left hand side in (3.12) is an equivalent expression for the Hausdorff distance $d_{\mathcal{H}}(\Theta(x), \Theta(y))$, and so (3.6) holds.

We formalize a few remarks concerning Proposition 3.3.

Remark 3.4. Property (b) of Proposition 3.3 is precisely the notion that the set valued map $\Theta \rightarrow \wp(\mathcal{S}(N))$ is uniformly upper semicontinuous (see Chapter 1 of Aubin and Cellina [1] for the elementary notions concerning set-valued maps, including their semi-continuity). Hence, Proposition 3.3 says that for elliptic maps the uniform upper semicontinuity of $\Theta$ as a set value map is equivalent to the uniform Hausdorff continuity of the function $\Theta$ taking values in the metric space $\left(\mathcal{K}(\mathcal{S}(N)), d_{\mathcal{H}}\right)$. Property (c) in terms of translations by multiples of the identity matrix is the form in which we will normally use the uniform continuity.

Uniform Hausdorff continuity of $\Theta$ passes to the dual map and extends to the boundary of $\Omega$ in a way that preserves the ellipticity of $\Theta$.

Proposition 3.5. Let $\Theta: \Omega \rightarrow \mathcal{E} \subset \mathcal{K}(\mathcal{S}(N))$ be an elliptic map. Then:

(a) $\Theta$ is uniformly Hausdorff continuous if and only is the dual map $\widetilde{\Theta} i s$;

(b) if $\Theta$ is uniformly Hausdorff continuous, then $\Theta$ extends to a uniformly Hausdorff continuous elliptic map on $\bar{\Omega}$.

Proof: For part (a), calculating duals in the formulation (3.8) yields

$$
x, y \in \Omega \text { with }|x-y|<\delta \Rightarrow \widetilde{\Theta}(y) \subset \widetilde{\Theta}(x)-\eta I \text { and } \widetilde{\Theta}(x) \subset \widetilde{\Theta}(y)-\eta I,
$$

where one uses the duality formulas (c) and (d) of Proposition 2.3 for elliptic sets. Hence $\widetilde{\Theta}$ is also uniformly Hausdorff continuous and has the same $\eta, \delta$ relation as $\Theta$.

For part (b), since $\Theta$ is uniformly continuous on the bounded set $\Omega$, $\Theta$ extends to a uniformly Hausdorff continuous map on $\bar{\Omega}$ in the standard way. In particular, for any $x_{0} \in \partial \Omega$ the limiting set $\Theta\left(x_{0}\right)$ is the unique limit in the complete metric space $\mathcal{K}(\mathcal{S}(N))$ of the Cauchy 
sequence $\left\{\Theta\left(x_{k}\right)\right\}_{k \in \mathbb{N}}$ where $\left\{x_{n}\right\}_{k \in \mathbb{N}} \subset \Omega$ is an arbitrary sequence converging to $x_{0}$ and one has

$$
d_{\mathcal{H}}\left(\Theta\left(x_{k}\right), \Theta\left(x_{0}\right)\right) \rightarrow 0 \text { as } k \rightarrow+\infty .
$$

One must show that the limit set $\Theta\left(x_{0}\right)$ is elliptic. By construction, each limiting set $\Theta\left(x_{0}\right)$ is closed and must be nonempty, since otherwise (3.5) would contradict the convergence (3.13). It remains only to show that each $\Theta\left(x_{0}\right)$ is not $\mathcal{S}(N)$ and satisfies the positivity property in (2.8). For the positivity property, one uses the fact that each $A \in \Theta\left(x_{0}\right)$ is a limit in $\mathcal{S}(N)$ of a sequence $\left\{A_{k}\right\}_{k \in \mathbb{N}}$ with $A_{k} \in \Theta\left(x_{k}\right)$. Hence for each $A \in \Theta\left(x_{0}\right)$ and each $P \in \mathcal{P}$ one has

$$
A+P=\lim _{k \rightarrow+\infty}\left(A_{k}+P\right) \text { with } A_{k}+P \in \Theta\left(x_{k}\right),
$$

and hence $\Theta\left(x_{0}\right)+\mathcal{P} \subset \Theta\left(x_{0}\right)$, as desired. Finally, to show that each $\Theta\left(x_{0}\right) \neq \mathcal{S}(N)$, it suffices to show that

$$
d_{\mathcal{H}}(\Theta, \mathcal{S}(N))=+\infty \text { for each elliptic set } \Theta .
$$

Indeed, if $\Theta\left(x_{0}\right)=\mathcal{S}(N)$ then applying (3.14) with $\Theta=\Theta\left(x_{k}\right)$ would contradict the convergence (3.13). To show that (3.14) holds, it suffices to show that $\Theta^{c}=\mathcal{S}(N) \backslash \Theta$ contains balls of arbitrarily large radius so that no finite enlargement of $\Theta$ can exhaust $\mathcal{S}(N)$. Complements of elliptic sets are open and proper and hence contain balls about some element $A_{0} \in-\left(\widetilde{\Theta}^{\circ}\right)$. Translation by a fixed element of $\mathcal{S}(N)$ preserves the ellipticity of $\Theta$ and hence one may assume that $A_{0}=0$. By the ellipticity of $\widetilde{\Theta}$ one has $-\widetilde{\Theta}-\mathcal{P} \subset-\widetilde{\Theta}$ and hence $(-\mathcal{P})^{\circ} \subset \Theta^{c}$. It is easy to verify that for each $t<0$ one has $N_{|t|}(t I) \subset(-\mathcal{P})^{\circ} \subset \Theta^{c}$.

Remark 3.6. This extension result will be useful for the applications to elliptic branches of (1.1). More precisely, we will often require control on the associated elliptic map up to the boundary, but we would prefer to impose any needed structural conditions on $F(x, A)$ only for $x \in \Omega$. See Proposition 5.4 for one such illustration.

We conclude this section with the basic properties of $\Theta$-subharmonic functions associated to uniformly continuous maps that will be needed in the proof of the comparison principle. Many of them are analogs of well-known properties for classical viscosity subsolutions for PDEs. In the abstract setting of elliptic maps, their validity depend on varying degrees of regularity in the map $\Theta$. This will be clarified in the proof which exploits the characterization given in Theorem 2.11. 
Proposition 3.7. Let $\Theta$ be a uniformly Hausdorff continuous elliptic map.

(1) (Maximum Property) $u, v \in \Theta \mathrm{SH}(\Omega) \Rightarrow \max \{u, v\} \in \Theta \mathrm{SH}(\Omega)$.

(2) (Affine Property) $u \in \Theta \mathrm{SH}(\Omega)$ and $a$ is affine $\Rightarrow u+a \in \Theta \mathrm{SH}(\Omega)$.

(3) (Decreasing Limits Property) If $\left\{u_{n}\right\}_{n \in \mathbb{N} \subset \Theta \operatorname{SH}(\Omega)}$ is a decreasing sequence, then $u:=\lim _{n \rightarrow+\infty} u_{n} \in \Theta \mathrm{SH}(\Omega)$.

(4) (Families Locally Bounded Above Property) Let $\mathcal{F} \subset \Theta \mathrm{SH}(\Omega)$ be a non empty family of functions which are locally uniformly bounded from above. Then the upper envelope $u:=\sup _{f \in \mathcal{F}} f$ has upper semicontinuous regularization ${ }^{2} u^{*} \in \Theta \mathrm{SH}(\Omega)$.

(5) (Uniform Translation Property) All sufficiently small translates of $u \in \Theta \mathrm{SH}(\Omega)$ have a fixed small quadratic perturbation which is $\Theta$-subharmonic on the domain of the translate. In particular, for each $\varepsilon>0$ if $\delta=\delta(\varepsilon)>0$ is chosen as in the formulation (3.8) of uniform Hausdorff continuity then

$$
u_{y ; \varepsilon}:=u(\cdot+y)+\frac{\varepsilon}{2}|\cdot|^{2} \in \Theta \operatorname{SH}\left(\Omega_{\delta}\right), \forall y \in B_{\delta}(0),
$$

where $\Omega_{\delta}:=\{x \in \Omega: \operatorname{dist}(x, \partial \Omega)>\delta\}$.

(6) (Existence of Bounded Subsolutions) There are bounded $\Theta$-subharmonic functions on $\Omega$; in particular, $\tau Q_{0}(\cdot):=\frac{\tau}{2}|\cdot|^{2}$ is a smooth and $\Theta, \widetilde{\Theta}$-subharmonic function for each large $\tau$.

If $\Theta$ is a constant elliptic map, then a stronger consequence than (5) follows, namely $u_{y}=u(\cdot-y) \in \Theta \mathrm{SH}\left(\Omega_{\delta}\right)$ for all $y \in B_{\delta}(0)$. This property plays a key role in [11] but may fail if $\Theta$ is not constant.

Proof: Properties (1) and (2) hold for arbitrary elliptic maps. Indeed, the Maximum Property (1) follows easily from the characterization formula (2.27) by using an argument by contradiction as employed in the proof of that theorem. The Affine Property (2) reduces to the claim that for each $x_{0} \in \Omega, w \in \operatorname{USC}(\Omega)$ and $a$ affine one has

$$
w \in \mathrm{SA}\left(x_{0}\right) \Rightarrow w+a \in \mathrm{SA}\left(x_{0}\right) .
$$

The implication (3.15) follows easily from the pointwise characterization (2.26) of $\mathrm{SA}\left(x_{0}\right)$.

To prove the property (3), it suffices to show that for each fixed $x_{0} \in \Omega$ and each fixed $v \in C^{2}(\Omega)$ with $D^{2} v\left(x_{0}\right) \in \Theta\left(x_{0}\right)$ one has

$$
w:=u+v \in \operatorname{SA}\left(x_{0}\right) .
$$

${ }^{2}$ We recall that $u^{*}(x):=\limsup _{r \rightarrow 0^{+}}\left\{u(y): y \in \Omega \cap \bar{B}_{r}\left(x_{0}\right)\right\}$ for each $x \in \Omega$. 
Fix a sequence $\left\{\varepsilon_{j}\right\}_{j \in \mathbb{N}}$ with $\varepsilon_{j} \searrow 0$ as $j \rightarrow+\infty$. By the continuity property (3.8) of $\Theta$ in $x_{0}$ there exists $\delta_{j}=\delta_{j}\left(\varepsilon_{j} / 2\right)$ such that for each $j \in \mathbb{N}$

$$
D^{2} v\left(x_{0}\right)+\frac{\varepsilon_{j}}{2} I \in \widetilde{\Theta}(x) \text { for each } x \in B_{\delta_{j}}\left(x_{0}\right) .
$$

Since $v \in C^{2}(\Omega)$, one can make $\left\|D^{2} v(x)-D^{2} v\left(x_{0}\right)\right\|$ small and hence

$$
D^{2} v(x)+\varepsilon_{j} I \in \widetilde{\Theta}(x) \text { for each } x \in B_{\delta_{j}}\left(x_{0}\right),
$$

where one reduces $\delta_{j}$ if need be. Since $u_{n} \in \Theta \mathrm{SH}(\Omega)$ it follows that

$$
w_{n, j}:=u_{n}+v+\varepsilon_{j} Q_{x_{0}} \in \mathrm{SA}\left(B_{\delta_{j}}\left(x_{0}\right)\right)=\widetilde{\mathcal{P}} \mathrm{SH}\left(B_{\delta_{j}}\left(x_{0}\right)\right), \quad n, j \in \mathbb{N},
$$

with $Q_{x_{0}}$ as defined in (2.31). For each $j$ fixed, $\left\{w_{n, j}\right\}_{n \in \mathbb{N}}$ is a decreasing sequence in $\widetilde{\mathcal{P}} \mathrm{SH}\left(B_{\delta_{j}}\left(x_{0}\right)\right)$ and hence

$$
w_{j}:=u+v+\varepsilon_{j} Q_{x_{0}} \in \mathrm{SA}\left(B_{\delta_{j}}\left(x_{0}\right)\right)=\widetilde{\mathcal{P}} \mathrm{SH}\left(B_{\delta_{j}}\left(x_{0}\right)\right), \quad j \in \mathbb{N},
$$

by the Decreasing Limits Property for the constant elliptic map $\widetilde{P}$ on the open set $B_{\delta_{j}}\left(x_{0}\right)$ (see Section 4 of [11]). We now argue by contradiction. If (3.16) were false, then there exists a triple $(\varepsilon, r, a)$ such that

$$
(w-a)\left(x_{0}\right)=0 \text { and }(w-a)(x) \leq-\varepsilon\left|x-x_{0}\right|^{2}, \forall x \in B_{r}\left(x_{0}\right)
$$

and by taking $J \in \mathbb{N}$ large enough to ensure $\varepsilon_{J} \leq \varepsilon$ one has

$$
\left(w_{J}-a\right)\left(x_{0}\right)=0 \text { and }\left(w_{J}-a\right)(x) \leq-\frac{\varepsilon}{2}\left|x-x_{0}\right|^{2}, \forall x \in B_{r}\left(x_{0}\right),
$$

and hence $w_{J} \notin \mathrm{SA}\left(x_{0}\right)$ which contradicts (3.19).

For the property (4), it suffices to show that for each fixed $x_{0} \in \Omega$ and each fixed $v \in C^{2}(\Omega)$ with $D^{2} v\left(x_{0}\right) \in \Theta\left(x_{0}\right)$ one has

$$
w^{*}:=u^{*}+v \in \operatorname{SA}\left(x_{0}\right),
$$

where $w^{*}(x)=(u+v)^{*}(x)$ for all $x \in \Omega$ by the continuity of $v$. We argue as in the proof of property (3) exploiting the continuity of $\Theta$ in $x_{0}$. Consider $\left\{\varepsilon_{j}\right\}_{j \in \mathbb{N}}$ with $\varepsilon_{j} \searrow 0$ as $j \rightarrow+\infty$ and $\delta_{j}=\delta_{j}\left(\varepsilon_{j} / 2\right)$ such that (3.17) holds. Consider the function

$$
w_{j}:=\sup _{f \in \mathcal{F}}\left(f+v+\varepsilon_{j} Q_{x_{0}}\right)=u+v+\varepsilon_{j} Q_{x_{0}}, \quad j \in \mathbb{N},
$$

where $f+v+\varepsilon_{j} Q_{x_{0}} \in \operatorname{SA}\left(B_{\delta_{j}}\left(x_{0}\right)\right)=\widetilde{\mathcal{P}} \mathrm{SH}\left(B_{\delta_{j}}\left(x_{0}\right)\right)$ for each $j \in \mathbb{N}$. Property (4) for the constant elliptic map $\widetilde{P}$ on the open set $B_{\delta_{j}}\left(x_{0}\right)$ yields (see Section 4 of [11]):

$$
\begin{aligned}
w^{*}+\varepsilon_{j} Q_{x_{0}}=u^{*}+v+\varepsilon_{j} Q_{x_{0}}=\left[w_{j}\right]^{*} & \in \mathrm{SA}\left(B_{\delta_{j}}\left(x_{0}\right)\right) \\
= & \widetilde{\mathcal{P}} \operatorname{SH}\left(B_{\delta_{j}}\left(x_{0}\right)\right), \quad j \in \mathbb{N} .
\end{aligned}
$$


If (3.20) were false, then there exists a triple $(\varepsilon, r, a)$ such that

$$
\left(w^{*}-a\right)\left(x_{0}\right)=0 \text { and }\left(w^{*}-a\right)(x) \leq-\varepsilon\left|x-x_{0}\right|^{2}, \forall x \in B_{r}\left(x_{0}\right)
$$

and by taking $J$ large enough to ensure $\varepsilon_{J} \leq \varepsilon$ one has

$$
\begin{array}{r}
\left(w^{*}+\varepsilon_{J} Q_{x_{0}}-a\right)\left(x_{0}\right)=0 \text { and }\left(w^{*}+\varepsilon_{J} Q_{x_{0}}-a\right)(x) \leq-\frac{\varepsilon}{2}\left|x-x_{0}\right|^{2}, \\
\forall x \in B_{r}\left(x_{0}\right),
\end{array}
$$

and hence $w_{J}^{*} \notin \mathrm{SA}\left(x_{0}\right)$ which contradicts (3.21).

We note that the arguments used for (3) and (4) do not use the uniformity of the continuity property (3.8). It would suffice to ask that there exists $\delta_{j}=\delta_{j}\left(\varepsilon_{j}, x_{0}\right)$ such that (3.17) holds. This is because the argument in purely local near each fixed $x_{0}$. On the other hand, uniform Hausdorff continuity is really used for properties (5) and (6).

For the Uniform Translation Property (5), with $\delta=\delta(\varepsilon)$ as in (3.8) for $\widetilde{\Theta}$, one needs to show that for each $x_{0} \in \Omega_{\delta}$ and $y \in B_{\delta}(0)$ fixed one has

$$
u_{y ; \varepsilon}+v \in \mathrm{SA}\left(x_{0}\right), \forall v \in C^{2}(\Omega) \text { with } D^{2} v\left(x_{0}\right) \in \widetilde{\Theta}\left(x_{0}\right) .
$$

Defining the test function $\hat{v}_{y ; \varepsilon}$ by $\hat{v}_{y ; \varepsilon}(x)=v(x-y)+\frac{\varepsilon}{2}|x-y|^{2}$ one has

$$
D^{2} \hat{v}_{y ; \varepsilon}\left(x_{0}+y\right)=D^{2} v\left(x_{0}\right)+\varepsilon I \in \widetilde{\Theta}\left(x_{0}\right)+\varepsilon I \subset \widetilde{\Theta}\left(x_{0}+y\right)
$$

by the uniform continuity of $\widetilde{\Theta}$. Since $u \in \Theta \operatorname{SH}\left(x_{0}+y\right)$ and $\hat{v}_{y ; \varepsilon} \in C^{2}(\Omega)$ satisfies (3.23), one has

$$
u+\hat{v}_{y ; \varepsilon} \in \mathrm{SA}\left(x_{0}+y\right)
$$

and hence

$$
u(\cdot+y)+\hat{v}_{y ; \varepsilon}(\cdot+y) \in \mathrm{SA}\left(x_{0}\right),
$$

since subaffinity is preserved by translations. The affirmation (3.24) is precisely the needed relation (3.22) by how $u_{y ; \varepsilon}$ and $\hat{v}_{y ; \varepsilon}$ are defined.

For the claim (6), it suffices to find $\tau>0$ such that $\tau I \in \Theta(x)$ for each $x \in \Omega$ since there would a corresponding $\widetilde{\tau}$ for $\widetilde{\Theta}$ which is also uniformly upper semicontinuous and taking $\max \{\tau, \widetilde{\tau}\}$ would then work for both maps by the positivity property in (2.8). By Proposition 3.5(b), $\Theta, \widetilde{\Theta}$ extend to uniformly continuous maps on $\bar{\Omega}$. Since $\Theta(y)$ is an elliptic set, for each $y \in \bar{\Omega}$ there exists $t_{y} \in \mathbb{R}$ such that

$$
t I \in \Theta(y) \text { for each } t \geq t_{y} .
$$

Indeed, pick $A_{y} \in \Theta(y)$ and choose $t_{y} \geq \lambda_{N}\left(A_{y}\right)$ to find $t_{y} I=A_{y}+$ $\left(t_{y} I-A_{y}\right) \in \Theta(y)+\mathcal{P} \subset \Theta(y)$. Using the uniform continuity of $\Theta$ with 
$\varepsilon=1$, there exists $\delta=\delta(1)>0$ such that

$$
\Theta(y)+I \in \Theta(x) \text { for each } x, y \in \bar{\Omega} \text { with }|x-y|<\delta .
$$

Cover $\bar{\Omega}$ compact with $\left\{B_{\delta}\left(y_{k}\right)\right\}_{k=1}^{n}$ having $y_{k} \in \bar{\Omega}$. Combining (3.25) and (3.26), one has $\tau I \in \Theta(x)$ for each $x \in \Omega$ for $\tau=1+\max _{1 \leq k \leq n} t_{y_{k}}$.

3.2. The semi-convex case for arbitrary elliptic maps. A key step in the proof of the comparison principle is to prove the subaffine theorem (3.2) in the special case that $u \in \Theta \mathrm{SH}(\Omega), v \in \widetilde{\Theta} \mathrm{SH}(\Omega)$ are also semi-convex functions. In this special case, $\Theta$ can be an arbitrary elliptic map since the proof for constant elliptic maps given in $[\mathbf{1 1}]$ carries over without difficulty. The main tool is a useful test for a semi-convex function to be subaffine (see Lemma 3.8), which in turn depends on a deep fact about convex functions known as Slodkowski's largest eigenvalue theorem. For completeness, we will give a brief discussion of these ingredients.

Slodkowski's theorem concerns lower bounds on the "largest eigenvalue" of the Hessian of locally convex function $\phi$ on a domain $\Omega$ and is stated in terms of the auxiliary function $K$ defined at points $x \in \Omega$ where $\phi$ is differentiable by the formula

$$
K(\phi, x):=\limsup _{\varepsilon \rightarrow 0} 2 \varepsilon^{-2} \sup _{|y|=1}\{\phi(x+\varepsilon y)-\phi(x)-\varepsilon\langle D \phi(x), y\rangle\}
$$

and $K(\phi, x):=+\infty$ otherwise. By Alexandroff's theorem, $\phi$ is twice differentiable for a.e. $x \in \Omega$ and at such points, $K(\phi, x)=\lambda_{N}\left(D^{2} \phi(x)\right)$. Slodkowski's theorem, as given in Corollary 3.5 of [22] states that for a locally convex function $\phi$ :

$$
K(v, \phi) \geq \Lambda \text { for a.e. } x \in \Omega \Rightarrow K(\phi, x) \geq \Lambda \text { for every } x \in \Omega .
$$

Slodkowski's theorem plays the role of Jensen's lemma in classical approaches to comparison theorems for viscosity solutions (see Lemma 3.10 of Jensen [16] and Lemma A.3 of [9]). In fact, these two results are in some sense equivalent as described in Harvey-Lawson [13]. A test for subaffinity of locally semi-convex functions follows from (3.28), as proven in Theorem 7.3 of $[\mathbf{1 1}]$.

Lemma 3.8. For u locally semi-convex on $\Omega$ one has

$$
D^{2} u(x) \in \widetilde{\mathcal{P}} \text { for a.e. } x \in \Omega \Rightarrow u \in \widetilde{\mathcal{P}} \mathrm{SH}(\Omega)=\mathrm{SA}(\Omega) .
$$

A useful version of the subaffine theorem now follows easily. Recall that a function $u: \Omega \rightarrow \mathbb{R}$ is said to be $\lambda$-semi-convex if $u+\lambda Q_{0}$ is a convex function, where $Q_{0}(x)=\frac{1}{2}|x|^{2}$ and $\lambda \in(0,+\infty)$. 
Proposition 3.9. Let $\Theta$ be any elliptic map. If $u$ and $v$ are $\lambda$-semiconvex then

$$
u \in \Theta \mathrm{SH}(\Omega), v \in \widetilde{\Theta} \mathrm{SH}(\Omega) \Rightarrow u+v \in \mathrm{SA}(\Omega) .
$$

For constant elliptic maps this is Corollary 7.4 of $[\mathbf{1 1}]$ and the proof is identical. Since $u+v$ is $2 \lambda$-semi-convex one has

$$
D^{2}(u+v)(x) \in \Theta(x)+\widetilde{\Theta}(x) \subset \widetilde{\mathcal{P}} \text { for a.e. } x \in \Omega,
$$

and hence $u+v \in \mathrm{SA}(\Omega)$ by Lemma 3.8 .

\subsection{The general case for uniformly continuous elliptic maps.}

We are now ready for the proof that the comparison principle holds for semicontinuous functions if the elliptic map is uniformly Hausdorff continuous.

Proof of Theorem 1.1: As shown in Proposition 3.1, it suffices to prove that for $u, v \in \operatorname{USC}(\bar{\Omega})$ one has

$$
u \in \Theta \mathrm{SH}(\Omega), v \in \widetilde{\Theta} \mathrm{SH}(\Omega) \Rightarrow u+v \in \mathrm{SA}(\Omega) .
$$

We note that $u$ and $v$ are bounded from above since they are upper semicontinuous on the compact set $\bar{\Omega}$.

Step 1: Reduce (3.30) to the case that $u, v$ are bounded from below.

Indeed, if $u, v$ are not bounded from below replace them by

$$
u_{m}:=\max \left\{u, \tau Q_{0}-m\right\} \text { and } v_{m}:=\max \left\{v, \tau Q_{0}-m\right\} \text { with } m \in \mathbb{N}
$$

where $Q_{0}(\cdot):=\frac{1}{2}|\cdot|^{2}$ and $\tau$ is large and fixed so that $\tau Q_{0}$ is a smooth and bounded function satisfying $D^{2}\left(\tau Q_{0}\right) \in \Theta(\Omega) \cap \widetilde{\Theta}(\Omega)$ (by the property (6) of Proposition 3.7). Hence for each $m \in \mathbb{N}$, the same claims hold for $\tau Q_{0}-m$.

One has that $u_{m}, v_{m}$ defined by (3.31) give decreasing sequences of upper semicontinuous functions which are bounded from below. By the Maximum Property (1) of Proposition 3.7, one has that $u_{m} \in \Theta \mathrm{SH}(\Omega)$ and $v_{m} \in \widetilde{\Theta} \mathrm{SH}(\Omega)$ for each $m \in \mathbb{N}$. If the subaffine theorem (3.30) holds for $u_{m}, v_{m}$ bounded from below, one has that $w_{m}:=u_{m}+v_{m}$ is a decreasing sequence in $\mathrm{SA}(\Omega)=\widetilde{\mathcal{P}} \mathrm{SH}(\Omega)$. Applying the Decreasing Limits Property (3) of Proposition 3.7 with the uniformly continuous (constant) elliptic map $\widetilde{\mathcal{P}}$ shows that the limit $w=u+v$ belongs to $\mathrm{SA}(\Omega)$. Hence (3.30) holds for general $u, v$, which completes Step 1. 
Step 2: Proof of (3.30) in the case that $u, v$ are bounded from below.

In order to pass from the special case of $u$ and $v$ semi-convex (for which (3.30) holds by Proposition 3.9) to the general case of $u$ and $v$ upper semicontinuous, we will make use of sup-convolution approximations which ensure the desired semi-convexity but which might spoil the needed $\Theta, \widetilde{\Theta}$-subharmonicity. The uniform Hausdorff continuity of $\Theta, \widetilde{\Theta}$ ensures that a suitable quadratic correction can be found and that one can pass to the limit.

To this end, for $u \in \operatorname{USC}(\Omega)$ and bounded on $\Omega$, for each $\varepsilon>0$ one defines the sup-convolution $u^{\varepsilon}$ by

$$
u^{\varepsilon}(x)=\sup _{z \in \mathbb{R}^{N}}\left\{u(x-z)-\frac{1}{\varepsilon}|z|^{2}\right\}, \quad \forall x \in \Omega,
$$

where one extends $u$ to be $-\infty$ outside of $\Omega$. The function defined in (3.32) satisfies the following well-known properties (cf. Theorem 8.2 of [11], for example):

$$
u^{\varepsilon} \text { decreases to } u \text { as } \varepsilon \rightarrow 0
$$

and

$$
u^{\varepsilon} \text { is } \frac{2}{\varepsilon} \text {-semi-convex. }
$$

For $u \in \Theta \mathrm{SH}(\Omega)$ bounded with $|u| \leq M$ on $\Omega$, consider the family of quadratic perturbations $u^{\varepsilon}(\cdot)+\eta|\cdot|^{2}$ with $\eta>0$ small.

Claim. For every $\eta>0$ there exists $\bar{\varepsilon}=\bar{\varepsilon}(\eta)>0$ such that

$$
u^{\varepsilon}(\cdot)+\eta|\cdot|^{2} \in \Theta \mathrm{SH}\left(\Omega_{\delta}\right), \forall \varepsilon \in(0, \bar{\varepsilon}(\eta)],
$$

where

$$
\delta:=\sqrt{2 \varepsilon M} \text { and } \Omega_{\delta}:=\{x \in \Omega: \operatorname{dist}(x, \partial \Omega)>\delta\} .
$$

Indeed, the Uniform Translation Property (5) of Proposition 3.7 says that for each $\eta>0$ there exists $\delta=\delta(\eta)>0$ such that

$$
u_{z, \eta}(\cdot):=u(\cdot-z)+\eta|\cdot|^{2} \in \Theta \operatorname{SH}\left(\Omega_{\delta}\right), \forall z \in B_{\delta}(0) .
$$

Moreover, as noted in the proof of Proposition 3.5, the $\eta, \delta$ relation is the same for the dual map $\widetilde{\Theta}$ and hence there is an analogous family $\left\{v_{z, \eta}\right\}_{z \in B_{\delta}(0)}$ associated to $v$.

Now, for $\varepsilon>0$, consider the following family of functions on $\Omega_{\delta}$

$$
\mathcal{F}:=\left\{u(\cdot-z)-\frac{1}{\varepsilon}|z|^{2}+\eta|\cdot|^{2}:|z|<\delta\right\} .
$$


One has $\mathcal{F} \subset \Theta \operatorname{SH}\left(\Omega_{\delta}\right)$ by (3.34) and the Affine Property (2) of Proposition 3.7. In addition, the collection is locally uniformly bounded from above. By property (4) of Proposition 3.7, the function defined by

$u_{\eta}^{\varepsilon}(x):=\sup _{|z|<\delta}\left\{u(x-z)-\frac{1}{\varepsilon}|z|^{2}+\eta|x|^{2}\right\}=\sup _{|z|<\delta}\left\{u(x-z)-\frac{1}{\varepsilon}|z|^{2}\right\}+\eta|x|^{2}$

admits an upper semicontinuous regularization $\left[u_{\eta}^{\varepsilon}\right]^{*}$ which lies in $\Theta \mathrm{SH}\left(\Omega_{\delta}\right)$. By suitably restricting the parameter $\varepsilon$, one can express $u_{\eta}^{\varepsilon}$ in terms of the sup-convolution $u^{\varepsilon}$; more precisely, for each $\eta>0$ one has the following identity on $\Omega_{\delta(\eta)}$ :

$$
u_{\eta}^{\varepsilon}(\cdot):=u^{\varepsilon}(\cdot)+\eta|\cdot|^{2} \text { for } \varepsilon \in(0, \bar{\varepsilon}(\eta)] \text { with } \bar{\varepsilon}(\eta)=\frac{\delta^{2}(\eta)}{2 M} .
$$

Indeed, with the restriction $\varepsilon \in\left(0, \delta^{2}(\eta) / 2 M\right)$ it is easy to see that values of $z$ with $|z| \geq \delta$ do not compete in the sup which defines $u^{\varepsilon}$ in (3.32). From (3.35) one has that $u_{\eta}^{\varepsilon}$ is semi-convex and hence continuous. Thus $u_{\eta}^{\varepsilon}=\left[u_{\eta}^{\varepsilon}\right]^{*} \in \Theta \operatorname{SH}\left(\Omega_{\delta}\right)$ and the claim (3.33) follows.

To complete Step 2, fix a sequence $\left\{\eta_{j}\right\}_{j \in \mathbb{N}}$ with $\eta_{j} \rightarrow 0$ as $j \rightarrow+\infty$ and select $\varepsilon_{j}:=\min \left\{\eta_{j}, \bar{\varepsilon}\left(\eta_{j}\right)\right\}$ so that $\delta_{j}:=\delta\left(\varepsilon_{j}\right)=\sqrt{2 \varepsilon_{j} M} \rightarrow 0^{+}$and $\Omega_{\delta_{j}} \nearrow \Omega$. The approximating sequences $\left\{u_{\eta_{j}}^{\varepsilon_{j}}\right\},\left\{v_{\eta_{j}}^{\varepsilon}\right\}$ defined by (3.35) are $2 / \varepsilon_{j}$-semi-convex and $\Theta, \widetilde{\Theta}$-subharmonic in $\Omega_{\delta_{j}}$. By Proposition 3.1 one has $w_{j}:=u_{\eta_{j}}^{\varepsilon_{j}}+v_{\eta_{j}}^{\varepsilon} \in \operatorname{SA}\left(\Omega_{j}\right)=\widetilde{\mathcal{P}} \mathrm{SH}\left(\Omega_{j}\right)$. By construction $w_{j} \searrow u+v$ and $\Omega_{\delta_{j}} \searrow \Omega$ and hence $u+v \in \mathrm{SA}(\Omega)$ by applying the Decreasing Limits Property (3) of Proposition 3.7 with respect to the constant map $\widetilde{\mathcal{P}}$. This completes Step 2 and hence the proof of Theorem 1.1.

\section{Boundary convexity and the Dirichet problem for ellipitc maps}

In this section, we implement Perron's method to give existence and uniqueness of solutions to the Dirichlet problem for $\Theta$-harmonic functions when $\Theta$ is a uniformly Hausdorff continuous elliptic map. Having already established the comparison principle, it remains to establish a class of admissible domains which will be given in terms of a suitable notion of strict boundary convexity with respect to $\Theta$ which ensures the existence of suitable barriers. In all that follows, $\Omega$ will be a bounded domain in $\mathbb{R}^{N}$ with $C^{2}$ boundary; that is, for each $x \in \partial \Omega$ there exists $\rho \in C^{2}\left(B_{r}(x)\right)$ for some $r>0$ such that

$$
\Omega \cap B_{r}(x)=\left\{y \in B_{r}(x): \rho(y)<0\right\} \text { and } D \rho \neq 0 \text { on } B_{r}(x) .
$$


4.1. Elliptic cones and boundary convexity. As mentioned in the introduction, the convexity notion is formulated in terms of elliptic cones associated to elliptic maps by the formula (1.12) as introduced in [11] for constant elliptic maps. Elliptic cones are called Dirichlet ray sets in $[\mathbf{1 1}]$ where the notion is systematically developed.

Definition 4.1. A set $\vec{\Theta}$ is an elliptic cone if $\vec{\Theta}$ is both an elliptic subset and a pointed cone in the sense that

$$
A \in \vec{\Theta} \Leftrightarrow t A \in \vec{\Theta} \text { for all } t \geq 0 .
$$

As shown in Section 5 of [11], the formula (1.12) defines an elliptic cone $\vec{\Theta}$ for every elliptic set $\Theta$. Moreover, since $\vec{\Theta} \in \mathcal{E}$, property (4.1) implies

$$
A \in \vec{\Theta}^{\circ} \Rightarrow t A \in \vec{\Theta}^{\circ} \text { for all } t>0
$$

Important examples of elliptic cones are

$$
\overrightarrow{\mathcal{P}}=\mathcal{P} \text { and } \overrightarrow{\widetilde{\mathcal{P}}}=\widetilde{\mathcal{P}}
$$

Moreover, these are extremal examples in the sense that for any elliptic cone $\vec{\Theta}$ one has (see Section 5 of $[\mathbf{1 1}]$ ):

$$
\mathcal{P} \subseteq \vec{\Theta} \subseteq \widetilde{P}
$$

Definition 4.2. Let $\vec{\Theta}$ be an elliptic cone. The boundary $\partial \Omega$ will be said to be strictly $\vec{\Theta}$-convex in $x \in \partial \Omega$ if there exists a local defining function $\rho$ for $\partial \Omega$ in $x$ such that

$$
\left.D^{2} \rho(x)\right|_{T_{x} \partial \Omega}=\left.B\right|_{T_{x} \partial \Omega} \text { for some } B \in \vec{\Theta}^{\circ},
$$

where $T_{x} \partial \Omega$ is the tangent space to $\partial \Omega$ at $x$.

As shown in Lemma 5.2 of [11], this convexity notion is independent of the choice of the local defining function $\rho$ where (4.2) plays a role in the proof. Since $\Theta \in \mathcal{E}$ determines $\vec{\Theta}$, for an elliptic map $\Theta: \bar{\Omega} \rightarrow \mathcal{E}$ one can define the elliptic cone map by using (1.12) pointwise; that is,

$$
\vec{\Theta}(x):=\vec{\Theta}(x) \text { for each } x \in \bar{\Omega} .
$$

If the elliptic map is uniformly Hausdorff continuous on $\Omega$, then both $\Theta$ and its dual $\widetilde{\Theta}$ extend as uniformly Hausdorff continuous elliptic maps on $\bar{\Omega}$ by Proposition 3.5. Hence the needed cones at the boundary will be determined by the values of the elliptic map near the boundary. Moreover, the uniform Hausdorff continuity implies that the cone map (4.4) is constant, as is the dual elliptic cone map $\overrightarrow{\widetilde{\Theta}}$ associated to $\widetilde{\Theta}$. 
Proposition 4.3. If $\Theta$ is a uniformly Hausdorff continuous elliptic map on $\Omega$ then there exists a fixed elliptic cone $\vec{\Theta}$ such that

$$
\vec{\Theta}(x)=\vec{\Theta} \text { for each } x \in \bar{\Omega} \text {. }
$$

In addition, $\vec{\Theta}=\Theta(x)$ for each $x \in \bar{\Omega}$ such that $\Theta(x)$ is itself an elliptic cone.

Proof: For the first claim, one makes use of the following characterization of the interior of the associated cone $\vec{\Theta}(x)$ (see Corollary 5.10 of [11]):

$$
\begin{aligned}
A \in \vec{\Theta}(x)^{\circ} \Leftrightarrow \exists \varepsilon>0 \text { and } R>0 \\
\text { such that } t(A-\varepsilon I) \in \Theta(x), \forall t \geq R .
\end{aligned}
$$

Moreover, if $A_{0} \in \Theta(x)^{\circ}$ then there exist $\varepsilon_{0}>0$ and $R_{0}>0$ such that (4.5) holds for each $A$ in a neighborhood of $A_{0}$ and each pair $R \geq R_{0}$, $\varepsilon \leq \varepsilon_{0}$. Now, with $x \in \bar{\Omega}$ fixed and $A \in \vec{\Theta}(x)^{\circ}$ the characterization (4.5) yields

$$
\exists \bar{t}=\bar{t}(A) \text { such that } t A \in \Theta(x) \text { for every } t \geq \bar{t} \text {. }
$$

In fact, one picks $\bar{t}(A)=R$ so that $t A=t A-\varepsilon t I+\varepsilon t I \in \Theta(x)$ for each $t \geq R$. By the uniform continuity of $\Theta$, for fixed $\varepsilon>0$ there exists $\delta=\delta(\varepsilon)>0$ such that

$$
t A+\varepsilon I \in \Theta(y) \text { if } y \in B_{\delta}(x) \cap \bar{\Omega} \text { and } t \geq \bar{t} .
$$

Hence one has $A \in \vec{\Theta}(y)$ by the definition (1.12). Since $A \in \vec{\Theta}(x)^{\circ}$ is arbitrary, by passing to the closure, one obtains

$$
\vec{\Theta}(x) \subset \vec{\Theta}(y) \text { for all } x, y \in \bar{\Omega} \text { such that }|x-y|<\delta \text {. }
$$

Inverting the roles of $x$ and $y$ shows that $\vec{\Theta}$ is locally constant on the connected set $\Omega$.

For the second assertion, suppose that $x \in \bar{\Omega}$ is such that $\Theta(x) \in \mathcal{E}$ is itself a cone; that is, if condition (4.1) holds, then

$$
\vec{\Theta}(x)=\Theta(x) \text {. }
$$

Indeed, one sees that $\Theta(x) \subset \vec{\Theta}(x)$ by taking $t_{0}=0$ in (1.12). Conversely, if $A_{0} \in \vec{\Theta}(x)$ then it is a limit in $\mathcal{S}(N)$ of $A_{k} \in \mathcal{S}(N)$ such that $t A_{k} \in \Theta(x)$ for each $t \geq t_{k}$ with $t_{k}=t_{0}\left(A_{k}\right)$. If $t_{k} \leq 1$ then $A_{k} \in \Theta(x)$. If, on the other hand $t_{k}>1$, then $t_{k} A_{k} \in \Theta(x)$ and using the cone property for $\Theta(x)$ one again has $A_{k} \in \Theta(x)$ by selecting $t=1 / t_{k}$. Hence the claim (4.7) follows since $\Theta(x)$ is closed. 
For a uniformly Hausdorff continuous map $\Theta$, one has a fixed elliptic cone $\vec{\Theta}$ to use for the formulation of boundary convexity in Definition 4.2 and the following result applies to give the existence of $\vec{\Theta}$-subharmonic global defining functions $\rho$ for the boundary; that is, $\rho \in C^{2}(\bar{\Omega})$ such that $\Omega=\{\rho<0\}, D \rho \neq 0$ on $\partial \Omega$ and $\rho$ is $\vec{\Theta}$-subharmonic on $\bar{\Omega}$.

Theorem 4.4. Let $\vec{\Theta}$ be an elliptic cone. If $\partial \Omega$ is strictly $\vec{\Theta}$-convex at each $x \in \partial \Omega$, there exists a global defining function $\rho$ for $\partial \Omega$ that is strictly $\vec{\Theta}$-subharmonic on $\bar{\Omega}$. In particular, if $\partial \Omega$ is strictly $\vec{\Theta}$-convex and $\vec{\Theta}$ is the elliptic cone associated to a uniformly Hausdorff continuous elliptic map $\Theta$ on $\bar{\Omega}$, then

(4.8) $\exists \varepsilon>0, R>0$ such that $C\left(\rho-\varepsilon|\cdot|^{2} / 2\right)$ is $\Theta$-subharmonic on $\Omega$

$$
\text { for all } C \geq R \text {. }
$$

Proof: When $\Theta$ is an elliptic set (a constant elliptic map), this is Theorem 5.12 of $[\mathbf{1 1}]$ and so we have the existence of $\rho$ since $\vec{\Theta}$ is constant by Proposition 4.3. When $\Theta$ is a non constant elliptic map, the proof of (4.8) requires some additional reasoning. By the characterization (4.6), for every $x \in \bar{\Omega}$ there exist $\varepsilon_{x}>0$ and $C_{x}>0$ such that

$$
C(A-3 \varepsilon I) \in \Theta(x)^{\circ}
$$

for all $C \geq C_{x}, \varepsilon \leq \varepsilon_{x}$ and $A$ in a neighborhood of $D^{2} \rho(x)$. Since $\rho \in \mathcal{C}^{2}(\bar{\Omega})$ one has that $C\left(D^{2} \rho(y)-2 \varepsilon I\right) \in \Theta(x)^{\circ}$ for every $y$ in a neighborhood $U_{x}$ of $x$. By the uniform continuity of $\Theta$ (and shrinking $U_{x}$, if necessary) one obtains $C\left(D^{2} \rho(y)-2 \varepsilon I\right)+C_{x} \varepsilon_{x} I \in \Theta(y)$ on $U_{x}$. Hence $C\left(D^{2} \rho(y)-\varepsilon I\right) \in \Theta(y)+C \varepsilon I-C_{x} \varepsilon_{x} I$. By extracting a finite subcovering $\left\{U_{x_{i}}\right\}_{i=1}^{N}$ of $\bar{\Omega}$, and by taking the minimum $\bar{\varepsilon}$ of $\left\{\varepsilon_{x_{i}}\right\}_{i=1}^{N}$ and the maximum $C$ of $\left\{C_{x_{i}}\right\}_{i=1}^{N}$, one obtains that $C\left(D^{2} \rho(y)-\bar{\varepsilon} I\right) \in$ $\Theta(y)+C \bar{\varepsilon} I-C_{x} \varepsilon_{x} I \subset \Theta(y)$ on $\bar{\Omega}$ with $C \gg 0$.

4.2. Perron's method and the Dirichlet problem. For a uniformly Hausdorff continuous map $\Theta$, we are now prepared to implement Perron's method to prove the existence and uniqueness of a $\Theta$-harmonic function with prescribed continuous boundary values.

Proof of Theorem 1.2: Uniqueness: This follows immediately from the comparison principle of Theorem 1.1. In fact, if $u, v$ are two solutions 
then $u \leq v$ and $v \leq u$ on $\partial \Omega$ where $u, v \in \Theta \operatorname{SH}(\Omega)$ and $-u,-v \in \widetilde{\Theta} \operatorname{SH}(\Omega)$ with $\Theta$ and $\widetilde{\Theta}$ uniformly Hausdorff continuous.

Existence: Consider the Perron family for the given boundary data $\varphi$

$$
\mathcal{F}(\varphi):=\left\{v \in \operatorname{USC}(\bar{\Omega}):\left.v\right|_{\Omega} \in \Theta \operatorname{SH}(\Omega) \text { and }\left.v\right|_{\partial \Omega} \leq \varphi\right\} .
$$

We will show that the upper envelope

$$
u(x):=\sup _{v \in \mathcal{F}(\varphi)} v(x), \quad \forall x \in \bar{\Omega},
$$

is continuous, $\Theta$-harmonic and satisfies the boundary condition.

Step 1: The Perron family (4.9) is uniformly bounded from above on $\bar{\Omega}$ and hence the upper envelope (4.10) is well defined and finite on $\bar{\Omega}$.

Property (6) of Proposition 3.7 ensures that the quadratic form $\tau Q_{0} \in$ $C^{2}(\bar{\Omega}) \cap \widetilde{\Theta} \mathrm{SH}(\Omega)$ for $\tau>0$ large enough and hence

$$
v+\tau Q_{0} \in \operatorname{USC}(\bar{\Omega}) \cap \operatorname{SA}(\Omega), \forall v \in \mathcal{F}(\varphi) .
$$

One then uses the maximum principle for subaffine functions (cf. Proposition 2.3 of $[\mathbf{1 1}])$ : if $w \in \operatorname{USC}(\bar{\Omega}) \cap \operatorname{SA}(\Omega)$ then

$$
\max _{\bar{\Omega}} w \leq \max _{\partial \Omega} w .
$$

Using (4.11), (4.12), and the non-negativity of $\tau Q_{0}$ one has

$$
v \leq v+\tau Q_{0} \leq \max _{\partial \Omega}\left(v+\tau Q_{0}\right) \leq \max _{\partial \Omega}\left(\varphi+\tau Q_{0}\right) \leq M \text { on } \bar{\Omega},
$$

for some $M \in \mathbb{R}$ as $\varphi$ are $Q_{\widetilde{B}}$ bounded.

Step 2: The upper envelope $u$ belongs to the Perron family $\mathcal{F}(\varphi)$.

When $\Theta$ is constant, this is Proposition 6.7 of [11] and the proof generalizes easily to the case of $\Theta$ uniformly Hausdorff continuous on $\bar{\Omega}$. Indeed, property (4) of Proposition 3.7 for the space $\Theta \mathrm{SH}(\Omega)$ shows that the upper semicontinuous regularization of $u$ satisfies

$$
u^{*} \in \Theta \operatorname{SH}(\Omega) \cap \operatorname{USC}(\bar{\Omega}) .
$$

It then suffices to show that

$$
u_{\mid \partial \Omega}^{*} \leq \varphi
$$

since then $u^{*} \in \mathcal{F}(\varphi)$ and hence $u^{*} \leq u$ on $\bar{\Omega}$, but $u \leq u^{*}$ on $\bar{\Omega}$ is always true. One uses a barrier argument to establish (4.13) where the uniform continuity of $\widetilde{\Theta}$ yields a smooth global defining function $\rho$ which is strictly $\overrightarrow{\widetilde{\Theta}}$-subharmonic on $\bar{\Omega}$ by Theorem 4.4. In particular, for each 
$x_{0} \in \partial \Omega$ fixed, combining the Affine Property (2) of Proposition 3.7 with (4.8) yields the existence of $\varepsilon>0$ and $R>0$ so that

$$
C\left(\rho-\varepsilon\left|\cdot-x_{0}\right|^{2}\right) \text { is } \widetilde{\Theta} \text {-subharmonic on } \Omega \text { for all } C \geq R .
$$

Since $\rho$ vanishes on $\partial \Omega$, for each $\delta>0$ one can pick $C$ in (4.14) large enough to ensure

$$
\begin{array}{r}
\varphi(x)+C\left(\rho-\varepsilon\left|x-x_{0}\right|^{2}\right)=\varphi(x)-C \varepsilon\left|x-x_{0}\right|^{2} \leq \varphi\left(x_{0}\right)+\delta \\
\text { for all } x \in \partial \Omega .
\end{array}
$$

By (4.14) and (4.15), for each $v \in \mathcal{F}(\varphi) \subset \operatorname{USC}(\bar{\Omega}) \cap \Theta \operatorname{SH}(\Omega)$ one has

$$
v+C\left(\rho-\varepsilon\left|\cdot-x_{0}\right|^{2}\right) \in \operatorname{USC}(\bar{\Omega}) \cap \mathrm{SA}(\Omega) \text { with } w_{\mid \partial \Omega} \leq \varphi\left(x_{0}\right)+\delta .
$$

Applying the maximum principle for subaffine functions (2.25) then gives

$$
v(x)+C\left(\rho-\varepsilon\left|x-x_{0}\right|^{2}\right) \leq \varphi\left(x_{0}\right)+\delta \text { for each } x \in \bar{\Omega} .
$$

Taking the sup over $v \in \mathcal{F}(\varphi)$ in (4.16) and regularizing yields

$$
u^{*}(x)+C\left(\rho-\varepsilon\left|x-x_{0}\right|^{2}\right) \leq \varphi\left(x_{0}\right)+\delta \text { for each } x \in \bar{\Omega},
$$

which when evaluated in $x=x_{0}$ yields the claim (4.13), as $x_{0}$ was arbitrary.

Step 3: $-u_{\mid \Omega} \in \widetilde{\Theta} \mathrm{SH}(\Omega)$.

One argues by contradiction and uses a "bump construction" which exploits the Maximum Property (1) of Lemma 3.7 (as done in Lemma 6.12 of $[\mathbf{1 1}]$ for $\Theta$ a constant elliptic map). Indeed, suppose that $-\left.u\right|_{\Omega} \notin \widetilde{\Theta} \mathrm{SH}(\Omega)$ and hence there exist $x_{0} \in \Omega$ and $A \in \widetilde{\widetilde{\Theta}}\left(x_{0}\right)=\Theta\left(x_{0}\right)$ such that $-u+\frac{1}{2}\left\langle A\left(x-x_{0}\right), x-x_{0}\right\rangle$ is not subaffine in $x_{0}$. For a suitable affine function $a$ and suitable $\varepsilon, r>0$ the function

$$
w= \begin{cases}u & \text { on } \bar{\Omega} \backslash B_{r}\left(x_{0}\right), \\ \max \left\{u, \frac{1}{2}\left\langle A\left(x-x_{0}\right), x-x_{0}\right\rangle+a+\varepsilon\left|x-x_{0}\right|^{2}\right\} & \text { on } B_{r}\left(x_{0}\right),\end{cases}
$$

satisfies $w\left(x_{0}\right)>u\left(x_{0}\right)$. However, $w$ belongs to the family $\mathcal{F}(\varphi)$ and hence satisfies $w\left(x_{0}\right) \leq u\left(x_{0}\right)$, a contradiction.

Step 4: $u$ is continuous at each point $x_{0} \in \partial \Omega$ and $\left.u\right|_{\partial \Omega}=\varphi$.

Since $u \in \operatorname{USC}(\bar{\Omega})$ and $\left.u\right|_{\partial \Omega} \leq \varphi$ by Step 1 , it suffices to show that

$$
\liminf _{x \rightarrow x_{0}} u(x) \geq \varphi\left(x_{0}\right) \text { for each } x_{0} \in \partial \Omega .
$$


This can be done with a barrier argument as in Step 2 which now exploits the assumption that $\partial \Omega$ is also strictly $\vec{\Theta}$-convex (see Lemma 6.9 of [11]) for constant elliptic maps).

Step 5: $u$ is continuous at each point $x \in \Omega$.

One exploits an argument of Walsh [24] as done for constant $\Theta$ in Proposition 6.11 of [11]. The proof must be adapted to the Uniform Translation Property (5) of Proposition 3.7 in place of the stronger translation property available in that special case (see the remark on property (5) preceding the proof of Proposition 3.7). Fix $\varepsilon>0$ and set $\bar{\varepsilon}=\varepsilon / d^{2}$ with $d:=\sup _{x \in \Omega}|x|$. By property (5), there exists $\delta>0$ such that for every $y \in B_{\delta}(0)$ one has

$u_{y}+\bar{\varepsilon}|\cdot|^{2}:=u(\cdot-y)+\bar{\varepsilon}|\cdot|^{2} \in \Theta \operatorname{SH}\left(\Omega_{\delta}\right)$ where $\Omega_{\delta}:=\{x \in \Omega: d(x, \partial \Omega)>\delta\}$, and we extend $u$ to be $-\infty$ on $\mathbb{R}^{n} \backslash \bar{\Omega}$. Let $C_{\delta}=\{x \in \bar{\Omega}: d(x, \partial \Omega)<\delta\}$ and since $u \in C(\partial \Omega)$, by rescaling $\delta$ if necessary, one has

$$
u_{y} \leq u+\varepsilon \text { on } C_{2 \delta} \text { if }|y|<\delta .
$$

Define the function $g_{y} \in \operatorname{USC}(\bar{\Omega})$ by

$$
g_{y}:=\max \left\{u_{y}+\bar{\varepsilon}|\cdot|^{2}-2 \varepsilon, u\right\},
$$

where $g_{y} \in \Theta \operatorname{SH}\left(\Omega_{\delta}\right)$ for each $y \in B_{\delta}(0)$ since the Affine Property (1) and the Maximum Property (2) of Proposition 3.7 can be applied. In addition, $g_{y}=u$ on $C_{2 \delta}$ by using (4.17) and the definition of $\bar{\varepsilon}$. Hence $g_{y} \in \mathcal{F}(\varphi)$ and so $g_{y} \leq u$ on $\bar{\Omega}$ as $u$ is the maximal element. Hence for each $y$ with $|y|<\delta$ one has

$$
u(x-y)+\bar{\varepsilon}|x|^{2}-2 \varepsilon \leq g_{y}(x) \leq u(x) \text { for each } x \in \bar{\Omega}
$$

and the change of variables $z=x+y$ yields

$$
\text { if } z, x \in \bar{\Omega} \text { and }|z-x|<\delta \text {, then } u(z) \leq u(x)+2 \varepsilon \text {. }
$$

Interchanging the roles of $x$ and $y$, by exploiting the symmetry in the definition of uniform continuity for $\Theta$, one obtains $|u(x)-u(z)| \leq 2 \varepsilon$.

\section{Applications to fully nonlinear PDEs}

In this section, we return to the nonlinear PDE (1.1) armed with the abstract existence result of Theorem 1.2 for elliptic maps $\Theta$. We will provide structural conditions on $F$ which ensure the existence of branches defined by uniformly Hausdorff continuous elliptic maps and give a description of the interiors of the associated elliptic cones which determine the needed boundary convexity. The general program will be illustrated with concrete examples which enable some comparison with the classical viscosity theory. 
5.1. Structure conditions, the comparison principle, and admissible domains. We begin by considering $F(x, A)$ which is monotone in $A$ along a given background elliptic map $\Phi$, which takes values in proper subsets of $\mathcal{S}(N)$. This is one way in which the abstract theory will be applied, but not the only one (see Remarks 5.8 and 5.14). The first result establishes the existence of an elliptic branch of a given PDE (1.1).

Proposition 5.1. Let $\Phi$ be an elliptic map on $\Omega$ and let $F \in C(\Omega \times$ $\mathcal{S}(N), \mathbb{R})$ be such that the conditions (1.14), (1.15), and (1.16) hold. Then the map $\Theta: \Omega \rightarrow \wp(\mathcal{S}(N))$ defined by (1.17) is an elliptic map and defines an elliptic branch of (1.1).

Proof: For each $x \in \Omega, \Theta(x) \neq \emptyset$ by (1.15) and is not all of $\mathcal{S}(N)$ since $\Phi(x)$ is an elliptic subset. Moreover $\Theta(x)$ is closed since $\Phi(x)$ is closed and $F$ is continuous. For each $x \in \Omega$ and for each $A \in \Theta(x) \subset \Phi(x)$ one has

$$
A+P \in \Phi(x), \quad \forall P \in \mathcal{P}
$$

and hence by (1.14) and (1.17) one has

$$
F(x, A+P) \geq F(x, A) \geq 0 .
$$

Hence $\Theta(x) \in \mathcal{E}$ for each $x$ and $\Theta$ is an elliptic map. This elliptic map will define an elliptic branch if (2.12) holds. One easily checks that

$$
\begin{aligned}
\partial \Theta(x)=[\partial \Phi(x) \cap\{A \in \mathcal{S}(N) & : F(x, A) \geq 0\}] \\
\cup & {[\Phi(x) \cap\{A \in \mathcal{S}(N): F(x, A)=0\}], }
\end{aligned}
$$

which yields (2.12) if (1.16) holds.

We have the following natural notion of viscosity solution for the natural branch $\Theta$ determined by $(F, \Phi)$ which uses $\Phi$ as an admissibility condition.

Definition 5.2. Let $F: \Omega \times \mathcal{S}(N) \rightarrow \mathbb{R}$ be continuous and $\Phi$ an elliptic map on $\Omega$.

(a) One says that $u \in \operatorname{USC}(\Omega)$ is a $\Phi$-admissible viscosity subsolution of (1.1) in $\Omega$ if for every $x_{0} \in \Omega$ and for each $\varphi \in C^{2}(\Omega)$ one has

(5.1) $u-\varphi$ has a local max in $x_{0}$

$$
\Rightarrow F\left(x_{0}, D^{2} \varphi\left(x_{0}\right)\right) \geq 0 \text { and } D^{2} \varphi\left(x_{0}\right) \in \Phi\left(x_{0}\right),
$$

where it is enough to consider $\varphi$ a quadratic function such that $\varphi\left(x_{0}\right)=u\left(x_{0}\right)$. 
(b) One says that $u \in \operatorname{LSC}(\Omega)$ is a $\Phi$-admissible viscosity supersolution of (1.1) in $\Omega$ if for every $x_{0} \in \Omega$ and for each $\varphi \in C^{2}(\Omega)$ one has

(5.2) $u-\varphi$ has a local min in $x_{0}$

$$
\Rightarrow F\left(x_{0}, D^{2} \varphi\left(x_{0}\right)\right) \leq 0 \text { or } D^{2} \varphi\left(x_{0}\right) \notin \Phi\left(x_{0}\right)^{\circ},
$$

where it is enough to consider $\varphi$ a quadratic function such that $\varphi\left(x_{0}\right)=u\left(x_{0}\right)$.

A $\Phi$-admissible viscosity solution of (1.1) in $\Omega$ is $u \in C(\Omega)$ which satisfies (a) and (b).

Notice that if one allows $\Phi\left(x_{0}\right)=\mathcal{S}(N)$, then the condition $D^{2} \varphi\left(x_{0}\right) \in$ $\Phi\left(x_{0}\right)$ in (5.1) is automatically satisfied while the condition $D^{2} \varphi\left(x_{0}\right) \notin$ $\Phi\left(x_{0}\right)$ in (5.2) is vacuous and one recovers the usual notions of viscosity sub and supersolutions (without restrictions). Furthermore, the condition $D^{2} \varphi\left(x_{0}\right) \notin \Phi\left(x_{0}\right)$ in (5.2) is natural as explained in Section V.3 of Ishii-Lions [15] in the special case of $\Phi \equiv \mathcal{P}$ for equations of MongeAmpère type.

We now examine the relation between the notion of $\Phi$-admissible viscosity solutions (Definition 5.2) and $\Theta$-harmonic maps (Definition 2.7) when the nonlinear PDE (1.1) admits an elliptic branch in accordance with Proposition 5.1.

Proposition 5.3. Let $F \in C(\Omega \times \mathcal{S}(N), \mathbb{R})$ and $\Phi: \Omega \rightarrow \mathcal{E}$ be such that (1.14) and (1.15) hold and let $\Theta$ be the corresponding elliptic map defined by (1.17). Then the following equivalences hold.

(a) $u \in \operatorname{USC}(\Omega)$ is a $\Phi$-admissible viscosity subsolution of (1.1) in $\Omega$ if and only if $u \in \Theta \mathrm{SH}(\Omega)$.

(b) $u \in \operatorname{LSC}(\Omega)$ is a $\Phi$-admissible viscosity supersolution of (1.1) in $\Omega$ if and only if $-u \in \widetilde{\Theta} \mathrm{SH}(\Omega)$ provided that the non-degeneracy condition (1.18) holds.

Condition (b) is part of formula (2.13) defining Krylov's notion of $F$ being a canonical form for $\Theta$.

Proof: In terms of the superdifferential (2.17), Definition 5.2(a) is equivalent to

$$
J^{+} u(x) \subset \Phi(x) \cap\{A \in \mathcal{S}(N): F(x, A) \geq 0\}=\Theta(x), \quad x \in \Omega,
$$

which is precisely (2.19) and we have part (a) of the equivalence.

Similarly, in terms of the subdifferential (2.18), Definition 5.2(b) is equivalent to

$$
J^{-} u(x) \subset\left[\Phi(x)^{\circ}\right]^{c} \cup\{A \in \mathcal{S}(N): F(x, A) \leq 0\}, \quad x \in \Omega .
$$


Since $J^{+}(-u)(x)=-J^{-} u(x)$ and $\widetilde{\Phi}(x)=-\left[\Phi(x)^{\circ}\right]^{c},(5.3)$ is equivalent to

$$
J^{+}(-u)(x) \subset \widetilde{\Phi}(x) \cup\{A \in \mathcal{S}(N): F(x,-A) \leq 0\}, \quad x \in \Omega .
$$

Hence it suffices to show that

$$
\widetilde{\Theta}(x)=\widetilde{\Phi}(x) \cup\{A \in \mathcal{S}(N): F(x,-A) \leq 0\}, \quad x \in \Omega .
$$

First, we calculate $\widetilde{\Theta}(x)$. By the definition (1.17), one has

$$
\begin{aligned}
\Theta(x) & =\{A \in \Phi(x): F(x, A) \geq 0\} \\
& =\Phi(x) \cap\{A \in \mathcal{S}(N): F(x, A) \geq 0\}, \quad x \in \Omega
\end{aligned}
$$

and using the property $[\widetilde{\Theta}(x)]^{\circ}=-[\Theta(x)]^{c}$ one finds

$$
[\widetilde{\Theta}(x)]^{\circ}=[\widetilde{\Phi}(x)]^{\circ} \cup\{A \in \mathcal{S}(N): F(x,-A)<0\},
$$

which by the property (2.3) yields

$$
\widetilde{\Theta}(x)=\widetilde{\Phi}(x) \cup \overline{\{A \in \mathcal{S}(N): F(x,-A)<0\}}, \quad x \in \Omega .
$$

By the continuity of $F$, from (5.6) we have the inclusion $\subset$ in (5.4). For the reverse inclusion in (5.4), it suffices to show that

$$
A \in \mathcal{S}(N) \text { with } F(x,-A) \leq 0 \Rightarrow A \in \widetilde{\Theta}(x) .
$$

By the non degeneracy condition (1.18), one has $-A \notin[\Theta(x)]^{\circ}$ and hence by duality one has $A \in \widetilde{\Theta}(x)$ as desired.

We now turn to structural conditions on $F$ which ensure the uniform Hausdorff continuity of the associated elliptic map and hence the validity of the comparison principle and to the proof of the general well posedness result of Theorem 1.3.

Proposition 5.4. Let $F \in C(\Omega \times \mathcal{S}(N), \mathbb{R})$ and let $\Phi$ be a uniformly Hausdorff continuous elliptic map on $\Omega$ such that the conditions (1.14), (1.15), and (1.19) hold. Then the elliptic map $\Theta$ defined by (1.17) extends to a uniformly Hausdorff continuous elliptic map on $\bar{\Omega}$.

Proof: $\Theta$ gives an elliptic map by Proposition 5.1 and will have the desired extension by Proposition 3.5, provided that $\Theta$ is uniformly Hausdorff continuous on $\Omega$. Given $\varepsilon^{*}>0$ so that (1.19) holds, the degenerate ellipticity (1.14) implies that (1.19) continues to hold for each $\varepsilon>\varepsilon^{*}$ by taking $\delta(\varepsilon)=\delta\left(\varepsilon^{*}\right)$. Hence for any $\varepsilon>0$, let $x, y \in \Omega$ with $|x-y|<\delta$ and take any $B \in \Theta(x)+\varepsilon I$ so that $B-\varepsilon I \in \Theta(x) \subset \Phi(x)$. Using the definition of $\Theta$ and (1.19) one finds

$$
0 \leq F(x, B-\varepsilon I) \leq F(y, B-\varepsilon I+\varepsilon I)=F(y, B),
$$


so that $B \in \Theta(y)$ provided that $B \in \Phi(y)$. Using the uniform continuity of $\Phi$, one has $B=(B-\varepsilon I)+\varepsilon I \in \Phi(y)$ for $x, y \in \Omega$ with $|x-y|<\delta_{\Phi}(\varepsilon)$. Hence $\Theta(x)+\varepsilon I \subset \Theta(y)$ for each $x, y \in \Omega$ with $|x-y|<\min \left\{\delta(\varepsilon), \delta_{\Phi}(\varepsilon)\right\}$. Interchanging the roles of $x$ and $y$ gives the uniform Hausdorff continuity of $\Theta$.

Proof of Theorem 1.3: $\Theta$ defines an elliptic branch of (1.1) by Proposition 5.1 and is uniformly Hausdorff continuous on $\Omega$ by Proposition 5.4. Hence the comparison principle of Theorem 1.1 holds for the map $\Theta$. Applying Theorem 1.2 yields a unique $u \in C(\bar{\Omega})$ which is $\Theta$-harmonic and satisfies $u=\varphi$ on $\partial \Omega$. Finally, $u$ is a $\Phi$-admissible viscosity solution of (1.1) by Proposition 5.3.

Finally, we give a description of the interiors of the elliptic cones $\vec{\Theta}^{\circ}$ and $\overrightarrow{\Theta^{\circ}}$ used to specify the needed strict convexity of admissible domains $\Omega$ for the Dirichlet problem.

Proposition 5.5. Let $\Phi, F$ be as in Proposition 5.4 and $\Theta$ the associated uniformly Hausdorff continuous elliptic map on $\bar{\Omega}$. Then, for any $\bar{x} \in \bar{\Omega}$,

$$
\vec{\Theta}^{\circ}=\left\{A \in \overrightarrow{\Phi(\bar{x}}^{\circ}: \exists \epsilon, R>0 \text { s.t. } F(\bar{x}, C(A-\epsilon I)) \geq 0, \forall C \geq R\right\}
$$

and

$$
\begin{aligned}
\overrightarrow{\Theta^{\circ}}=\overrightarrow{\widetilde{\Phi}}(\bar{x})^{\circ} \cup\{A \in \mathcal{S}(N): \exists \epsilon, R>0 \\
\text { s.t. } F(\bar{x}, C(-A+\epsilon I))<0, \forall C \geq R\},
\end{aligned}
$$

where $\overrightarrow{\Phi(x)}$ (resp. $\overrightarrow{\widetilde{\Phi}(x)})$ is the elliptic cone associated to $\Phi(x)$ (resp. $\widetilde{\Phi}(x))$.

Proof: We will use the following fact. For each $\Phi \in \mathcal{E}$ the following are equivalent:

(a) $A \in \vec{\Phi}^{\circ}$.

(b) There exist $\epsilon, R>0$ such that $C(A-\epsilon I) \in \Phi$ for all $C \geq R$.

(c) There exist $\hat{\epsilon}, R>0$ such that $C(A-\hat{\epsilon} I) \in \Phi^{\circ}$ for all $C \geq R$.

The equivalence between (a) and (b) is proved in Corollary 5.10 of [11] and (c) easily implies (b). Suppose now that (b) holds and let $\epsilon, R>0$ be such that $C(A-\epsilon I) \in \Phi$ for all $C \geq R$. Then, $C(A-\epsilon / 2 I)=$ $C(A-\epsilon I)+C \epsilon / 2 I \in \Phi+\mathcal{P}^{\circ} \subseteq \Phi^{\circ}$ for all $C \geq R>0$, which is (c).

By means of Proposition 4.3, the associated elliptic cone maps $\vec{\Theta}, \vec{\Theta}$ are constant in $\bar{\Omega}$, so $\vec{\Theta}=\overrightarrow{\Theta(\bar{x})}$ and $\overrightarrow{\widetilde{\Theta}}=\overrightarrow{\widetilde{\Theta}(\bar{x})}$ for any $\bar{x} \in \bar{\Omega}$. Hence, 
by (b) one concludes that

$$
\begin{aligned}
\vec{\Theta}^{\circ}=\overrightarrow{\Theta(\bar{x}}^{\circ}=\{A \in \mathcal{S}(N): \exists \epsilon, R>0 \text { s.t. } C(A-\epsilon I) \in \Phi(\bar{x}) \\
\quad \text { and } F(\bar{x}, C(A-\epsilon I)) \geq 0, \forall C \geq R\} \\
=\left\{A \in \overrightarrow{\Phi(\bar{x}}^{\circ}: \exists \epsilon, R>0 \text { s.t. } F(\bar{x}, C(A-\epsilon I)) \geq 0, \forall C \geq R\right\} .
\end{aligned}
$$

For $\overrightarrow{\widetilde{\Theta}^{\circ}}$, one uses $(\mathrm{c})$ and the identity $\widetilde{\Theta}(\bar{x})^{\circ}=\widetilde{\Phi}(\bar{x})^{\circ} \cup\{A \in \mathcal{S}(N)$ : $F(\bar{x},-A)<0\}$, which follows from (5.5). In fact, one finds

$$
\begin{aligned}
& \overrightarrow{\widetilde{\Theta}}^{\circ}=\overrightarrow{\widetilde{\Theta}(\bar{x})^{\circ}}=\left\{A \in \mathcal{S}(N): \exists \epsilon, R>0 \text { s.t. } C(A-\epsilon I) \in \widetilde{\Phi}(\bar{x})^{\circ}\right. \\
& \text { or } F(\bar{x},-C(A-\epsilon I))<0, \forall C \geq R\} \\
& =\overrightarrow{\widetilde{\Phi}}(\bar{x})^{\circ} \cup\{A \in \mathcal{S}(N): \exists \epsilon, R>0 \\
& \text { s.t. } F(\bar{x}, C(-A+\epsilon I))<0, \forall C \geq R\} \text {. }
\end{aligned}
$$

The following example gives a class of equations for which the above considerations hold, where $\mathrm{UC}(\Omega, \mathbb{R})$ is the space of uniformly continuous real valued functions on $\Omega$.

Example 5.6. Let $\Phi \in \mathcal{E}, f \in \mathrm{UC}(\Omega, \mathbb{R})$ and $G \in C(\mathcal{S}(N), \mathbb{R})$ be such that the following three conditions hold:

$$
G(A) \geq G(B) \text { for each } A, B \in \Phi \text { such that } A \geq B,
$$

$$
\forall x \in \Omega \text { there exists } A \in \Phi \text { such that } G(A)=f(x),
$$

and there exists $r^{*}>0$ so that

$$
G(A+r I) \geq G(A)+\beta(r) \text { for each } r \in\left(0, r^{*}\right] \text { and each } A \in \Phi
$$

for some function $\beta:\left(0, r^{*}\right] \rightarrow(0,+\infty)$. Then the function $F \in C(\Omega \times$ $\mathcal{S}(N), \mathbb{R})$ defined by

$$
F(x, A):=G(A)-f(x), \quad x \in \Omega, A \in \mathcal{S}(N)
$$

satisfies the hypotheses of Proposition 5.4 as is easily shown. Conditions which ensure (5.9) are not difficult to find since $G(\Phi)$ is connected. If $\Phi$ itself is an elliptic cone, one can express the needed cones (5.7) and (5.8) in terms of $G$ and the distance function to the boundary which will be of class $C^{2}$ if $\Omega$ is (see Foote $[\mathbf{1 0}]$ ).

Remark 5.7. Condition (5.10) resembles the non-totally degenerate condition of Bardi-Mannucci [2] which is used to prove the comparison 
principle for equations which can also depend on $(u, D u)$. For equations without this dependence their condition is

$\exists \eta>0$ such that $F(x, A+r I)-F(x, A) \geq \eta r$ for each $r>0$, which is (5.10) for $F(x, A)=G(A)-f(x)$ and a linear $\beta(r)=\eta r$. For this reason, we will refer to (5.10) (as well as the more general (1.19)) as a non-total degeneracy condition and they give alternatives to the standard structural condition placed on $F$ in order to obtain the comparison principle (see Remark 5.10 below).

Remark 5.8. If $F(x, A)$ is increasing in $A$ on all of $\mathcal{S}(N)$ the abstract theory still applies and corresponds to the choice $\Phi(x)=\mathcal{S}(N)$ in the results above. In particular, if $F$ is continuous and satisfies (1.15) then

$$
\Theta(x):=\{A \in \mathcal{S}(N): F(x, A) \geq 0\}, \quad x \in \Omega
$$

will satisfy all of the conditions for being an elliptic map except perhaps $\Theta(x) \neq \mathcal{S}(N)$, which can be checked in the applications. One then easily checks that $\Theta$ defined by (5.11) satisfies the conclusions of Propositions 5.1 and 5.4. Moreover, using $\vec{\Phi}=\mathcal{S}(N)$, one has the characterizations of $\vec{\Theta}^{\circ}, \overrightarrow{\Theta^{\circ}}$ of Proposition 5.5. Finally, the notion of being a $\Phi$-admissibile viscosity solution of $F=0$ reduces to being an admissible viscosity solution in the sense of Definition 2.7.

5.2. Solvability theorems for concrete examples. We begin with the perturbed Monge-Ampère equation (1.3) previously introduced which fits into the scheme of Propositions 5.4 and 5.5.

Theorem 5.9. Let $\Omega \subset \mathbb{R}^{N}$ be a bounded and strictly convex domain with $\partial \Omega$ of class $C^{2}$. Let $f \in \mathrm{UC}(\Omega, \mathbb{R})$ be non-negative and $M \in$ $\mathrm{UC}(\Omega, \mathcal{S}(N))$. Consider

$$
F(x, A):=\operatorname{det}(A+M(x))-f(x)
$$

and define

$\Phi(x):=\{A \in \mathcal{S}(N): A+M(x) \geq 0\}$ and $\Theta(x):=\{A \in \Phi(x): F(x, A) \geq 0\}$.

For each $\varphi \in C(\partial \Omega)$, there exists a unique $u \in C(\bar{\Omega})$ which is a $\Phi$-admissible viscosity solution of $F\left(x, D^{2} u(x)\right)=0$ in the sense of Definition 5.2 and satisfies $u=\varphi$ on $\partial \Omega$.

Proof: $\Phi$ is clearly elliptic since each $\Phi(x)=\mathcal{P}-M(x)$ is a translate in $\mathcal{S}(N)$ of the elliptic set $\mathcal{P}$ and the uniform continuity of $M$ yields

$$
A+\varepsilon I+M(y) \geq A+M(x) \geq 0 \text { for each } A \in \Phi(x) \text { and } x, y \in \Omega
$$

$$
\text { with }|x-y|<\delta_{M}(\varepsilon) \text {; }
$$


that is,

$$
\Phi(x)+\varepsilon I \subset \Phi(y) \text { for each } x, y \in \Omega \text { with }|x-y|<\delta_{M}(\varepsilon) .
$$

Interchanging the roles of $x$ and $y$ gives the uniform Hausdorff continuity of $\Phi$.

Clearly $F$ is continuous and satisfies (1.14) with respect to $\Phi$. For the property (1.15), notice that for each $x \in \Omega$ the range of

$$
\operatorname{det}(A+M(x)): \Phi(x) \rightarrow \mathbb{R}
$$

is the interval $[0,+\infty)$ since $\Phi(x)$ is connected, 0 is attained by $A=$ $-M(x) \in \Phi(x)$, and $\operatorname{det}(t I+M(x)) \rightarrow+\infty$ as $t \rightarrow+\infty$. By Proposition 5.4, $\Theta$ extends to a uniformly Hausdorff continuous elliptic map on $\bar{\Omega}$ provided that (1.19) holds (for each small $\varepsilon>0$ ). This follows from the uniform continuity of $f$ and $M$ since

$$
\begin{aligned}
F(y, A+\varepsilon I)-F(x, A)= & \operatorname{det}(A+\varepsilon I+M(y)) \\
& -\operatorname{det}(A+M(x))+f(x)-f(y) \\
\geq & \operatorname{det}(A+\varepsilon I+M(y)) \\
& -\operatorname{det}(A+M(x))-\omega(|x-y|),
\end{aligned}
$$

which will be non-negative if $|x-y|<\delta(\varepsilon)$ for a suitable $\delta$ involving the modulus of continuity of $M$.

Theorem 1.2 then gives the desired unique $\Theta$-harmonic solution on each admissible domain $\Omega$. Using the definition (1.12) one easily calculates the cone $\vec{\Phi}=\mathcal{P}$ from which it follows that $\vec{\Theta} \subset \mathcal{P}$ but $\mathcal{P} \subset \vec{\Theta}$ for any elliptic cone and hence $\mathcal{P}=\vec{\Theta}$. Thus the admissibility is just strict convexity of $\partial \Omega$ in the usual sense in light of (2.23) since $\vec{\Theta} \cap \overrightarrow{\widetilde{\Theta}}=\mathcal{P}$. Finally, $F$ clearly satisfies the non-degeneracy condition (1.18) and so Proposition 5.3 applies to complete the last claim.

Remark 5.10. As noted in the introduction, classical structural conditions used to prove the validity of the comparison principle may fail in cases where (1.19) holds. More precisely, condition (3.14) of CrandallIshii-Lions [9] (rewritten for $F(x, A)$ which is increasing in $A$ ) is the following: there exists $\omega:[0, \infty] \rightarrow[0, \infty]$ such that $\omega\left(0^{+}\right)=0$ and for each $\alpha>0$

$$
F(x, A)-F(y, B) \leq \omega\left(\alpha|x-y|^{2}+|x-y|\right)
$$

whenever $x, y \in \Omega$ and $A, B \in \mathcal{S}(N)$ satisfy

$$
-3 \alpha\left(\begin{array}{cc}
I & 0 \\
0 & I
\end{array}\right) \leq\left(\begin{array}{cc}
A & 0 \\
0 & -B
\end{array}\right) \leq 3 \alpha\left(\begin{array}{cc}
I & -I \\
-I & I
\end{array}\right) .
$$


For example, let $\Omega \subset \mathbb{R}^{2}$ be any smooth bounded domain containing the origin and consider $F(x, A)=\operatorname{det}(A+M(x))-f(x)$ with $f \in \mathrm{UC}(\Omega, \mathbb{R})$ non-negative and

$$
M(x):=\left(\begin{array}{cc}
g(x) & 0 \\
0 & 0
\end{array}\right), \text { with } g \in \mathrm{UC}(\Omega, \mathbb{R}) .
$$

While $F$ satisfies (1.19) for all $g \in \mathrm{UC}(\Omega, \mathbb{R})$, the conditions (5.12)-(5.13) require that if $g$ vanishes at an isolated point, it cannot vanish to order less than two. Indeed, assume that $g$ satisfies

$$
g(x)=0 \Leftrightarrow x=0 \text { and } \lim _{x \rightarrow 0} \frac{|x|^{2}}{|g(x)|}=0 .
$$

If an $\omega$ satisfying (5.12)-(5.13) were to exist, by taking $\left\{x_{n}\right\}_{n \in \mathbb{N}} \subset \Omega \backslash\{0\}$ such that $x_{n} \rightarrow 0$ as $n \rightarrow+\infty$ and setting

$$
A_{n}:=\left(\begin{array}{cc}
0 & 0 \\
0 & \frac{1}{2\left|g\left(x_{n}\right)\right|}
\end{array}\right), \quad B_{n}:=\left(\begin{array}{cc}
0 & 0 \\
0 & \frac{1}{\left|g\left(x_{n}\right)\right|}
\end{array}\right),
$$

one easily checks that $A_{n}, B_{n}$ satisfy (5.13) with $3 \alpha_{n}=1 /\left|g\left(x_{n}\right)\right|$. However, by (5.14) one has

$$
\begin{aligned}
\frac{1}{2}=F\left(x_{n}, A_{n}\right)-F\left(0, B_{n}\right)+ & f\left(x_{n}\right)-f(0) \\
& \leq \omega\left(\frac{\left|x_{n}\right|^{2}}{3\left|g\left(x_{n}\right)\right|}+\left|x_{n}\right|\right)+\omega_{f}\left(\left|x_{n}\right|\right) \rightarrow 0
\end{aligned}
$$

as $n \rightarrow \infty$, which leads to a contradiction if (5.12) were to hold.

The following result shows that no convexity/concavity with respect to $A$ is required for $F(x, A)$ and illustrates an application of the theory when $F$ is non-decreasing in $A$ on all of $\mathcal{S}(N)$. For $k=1, \ldots, N$, set:

$$
\mathcal{P}_{k \wedge(N-k+1)}:=\left\{A \in \mathcal{S}(N): \lambda_{k}(A) \geq 0 \text { and } \lambda_{N-k+1}(A) \geq 0\right\} .
$$

Theorem 5.11. Fix $k \in\{1, \ldots, N\}$ and $\Omega \subset \mathbb{R}^{N}$ a bounded and strictly $\mathcal{P}_{k \wedge(N-k+1)}$-convex domain with $\partial \Omega$ of class $C^{2}$ and let $f \in \mathrm{UC}(\Omega, \mathbb{R})$. Consider

$$
F_{k}(x, A):=\lambda_{k}(A)-f(x)
$$

and define

$$
\Theta_{k}:=\Theta_{k}(x):=\left\{A \in \mathcal{S}(N): \lambda_{k}(A)-f(x) \geq 0\right\} .
$$

For each $\varphi \in C(\partial \Omega)$, there exists a unique $u \in C(\bar{\Omega})$ which is a viscosity solution of $F_{k}\left(x, D^{2} u(x)\right)=0$ in the standard sense and satisfies $u=\varphi$ on $\partial \Omega$. 
Proof: Notice that $\Theta_{k}$ is clearly a proper subset of $\mathcal{S}(N)$ for each $x \in \Omega$ and hence, as explained in Remark 5.8, the general results of Subsection 5.1 apply. In particular, $\left.F_{k}(x, A):=G_{(} A\right)-f(x)$ where $G_{k}$ is nonincreasing in $A$ for $A \in \mathcal{S}(N)$ and satisfies (5.9) so that $\Theta_{k}$ is an elliptic map. One has $G_{k}(A+r I)=G_{k}(A)+r$ and hence the non-totally degenerate condition (5.10) holds with $\beta(r)=r$ and $\Theta_{k}$ is uniformly Hausdorff continuous. The remainder of the proof proceeds as that of Theorem 5.9 where one needs only to check the form of the elliptic cones used for the boundary convexity by using Proposition 5.5. For any $\bar{x} \in \Omega$,

$$
\begin{aligned}
\overrightarrow{\Theta_{k}} \circ & =\left\{A \in \mathcal{S}(N): \exists \epsilon, R>0 \text { s.t. } \lambda_{k}(C(A-\epsilon I))-f(\bar{x}) \geq 0 \text { for all } C \geq R\right\} \\
& =\left\{A \in \mathcal{S}(N): \exists \epsilon, R>0 \text { s.t. } \lambda_{k}(A) \geq \epsilon+f(\bar{x}) C^{-1} \text { for all } C \geq R\right\} \\
& =\left\{A \in \mathcal{S}(N): \lambda_{k}(A)>0\right\}=\mathcal{P}_{k}^{\circ} .
\end{aligned}
$$

The equality $\vec{\Theta}_{k}^{\circ}=\mathcal{P}_{N-k+1}^{\circ}$ can be verified in an analogous way.

Remark 5.12. As claimed above, $F_{k}(x, A)$ may be fail to be concave (resp. convex) in $A$. For example, if $N=3, k=2$, and $f(x)=0$ for some $x \in \Omega$ then $\Theta_{2}(x)=\left\{A \in \mathcal{S}(3): \lambda_{2}(A) \geq 0\right\}$. By (2.14) it suffices to check that $\Theta_{2}(x)$ (resp. $\left[\Theta_{2}(x)^{\circ}\right]^{c}$ ) are not convex and this is the case as one can see by considering the segments joining $A_{1}$ to $\pm A_{2}$ where

$$
A_{1}=\left[\begin{array}{rrr}
-1 & 0 & 0 \\
0 & 0 & 0 \\
0 & 0 & 0
\end{array}\right] \text { and } A_{2}=\left[\begin{array}{rrr}
0 & 0 & 0 \\
0 & -1 & 0 \\
0 & 0 & 0
\end{array}\right]
$$

We now consider a simple situation in which $F$ presents multiple branches and for which $F$ may not be monotone along the elliptic map.

Theorem 5.13. Let $\Omega \subset \mathbb{R}^{2}$ be a bounded and strictly convex domain with $\partial \Omega$ of class $C^{2}$ and let $f \in \mathrm{UC}(\Omega, \mathbb{R})$ be a non-negative function. Consider

$$
F(x, A):=A_{11} A_{22}-f(x)
$$

and for each $x \in \Omega$ define

$\Phi_{1}:=\left\{A \in \mathcal{S}(2): \min \left\{A_{11}, A_{22}\right\} \geq 0\right\}, \Theta_{1}(x):=\left\{A \in \Phi_{1}: F(x, A) \geq 0\right\}$, and

$$
\begin{aligned}
\Theta_{2}(x):=\left\{A \in \mathcal{S}(2): \max \left\{A_{11}, A_{22}\right\} \geq 0\right. \text { or } \\
{\left.\left[\max \left\{A_{11}, A_{22}\right\}<0 \text { and } F(x, A) \leq 0\right]\right\} . }
\end{aligned}
$$

For each $\varphi \in C(\partial \Omega)$ there exist unique elements $u_{1}, u_{2} \in C(\bar{\Omega})$ such that 
(a) $u_{1}$ is a $\Phi_{1}$-admissible viscosity solution of $F\left(x, D^{2} u(x)\right)=0$ in the sense of Definition 5.2 and satisfies $u_{1}=\varphi$ on $\partial \Omega$;

(b) $u_{2}$ is an admissible viscosity solution of the branch of $F\left(x, D^{2} u(x)\right)=0$ determined by $\Theta_{2}$ in the sense of Definition 2.7 and satisfies $u_{2}=\varphi$ on $\partial \Omega$.

Proof: For part (a), the constant map $\Phi_{1}$ is clearly elliptic and hence uniformly Hausdorff continuous. One easily checks that $F(x, A)$ is increasing along $A \in \Phi_{1}$ with zero locus intersecting $\Phi_{1}$ for each $x$ and hence $\Theta_{1}$ is an elliptic map by Proposition 5.1. For the uniform continuity of $\Theta_{1}$, one uses Proposition 5.4 where $F(x, A):=G(A)-f(x)$ with $f$ uniformly continuous and $G(A+r I)=G(A)+r\left(A_{11}+A_{22}\right)+r^{2}$ so that the non-total degeneracy condition (5.10) holds with $\beta(r)=r^{2}$ with $A \in \Phi_{1}$. In order to determine the admissible domains, using the definition of the boundary cones (1.12) and duality (2.5) one finds that

$$
\overrightarrow{\Theta_{1}}=\Phi_{1}=\overrightarrow{\Phi_{1}} \quad \text { and } \quad \widetilde{\Theta}_{1}(x)=\Theta_{2}(x), x \in \Omega .
$$

It follows that

$$
\overrightarrow{\Theta_{2}}=\overrightarrow{\widetilde{\Theta_{1}}}=\widetilde{\overrightarrow{\Theta_{1}}}=\widetilde{\Phi_{1}}=\Phi_{2}:=\left\{A \in \mathcal{S}(2): \max \left\{A_{11}, A_{22}\right\} \geq 0\right\} .
$$

Hence $\Omega$ must be both strictly $\overrightarrow{\Phi_{1}}, \overrightarrow{\Phi_{2}}$-convex, which then implies the usual notion of strict convexity with respect to $\mathcal{P}$. Theorem 1.2 then provides a unique $u_{1} \in C(\bar{\Omega})$ which is $\Theta_{1}$-harmonic and satisfies the boundary condition. Since the non-degeneracy condition (1.18) on $F$ holds, Proposition 5.3 gives that $u_{1}$ is a $\Phi_{1}$-admissible viscosity solution.

For part (b), the second formula in (5.15) says that $\Theta_{2}$ is the dual of $\Theta_{1}$ and hence $\Theta_{2}$ is also uniformly Hausdorff continuous and $\partial \Omega$ will have the same boundary convexity requirement. Theorem 1.2 yields the unique $u_{2} \in C(\bar{\Omega})$ which is $\Theta_{2}$-harmonic on $\Omega$ and satisfies the boundary condition. The branch condition (2.12) is clearly satisfied and hence $u_{2}$ is and admissible viscosity solution in the sense of Definition 2.7.

Remark 5.14. For $F(x, A)=A_{11} A_{22}-f(x)$, if $f(x)>0$, the zero locus $\Gamma(x)$ is made up of two hyperboloids which are are contained in the subsets of $\mathcal{S}(2)$ on which $A_{11}, A_{22}$ are positive and negative respectively. In the second case, one loses the monotonicity of $F$ along $\Theta_{2}$ and hence the equation will not be degenerate elliptic and moreover, since $F(x, A)<0$ in the interior of $\Theta_{2}$ near the zero locus, one cannot interpret $u_{2}$ as a viscosity solution of the PDE (1.1) in a natural way, although is a perfectly natural viscosity solution of the differential inclusion (2.11) defined by the elliptic branch $\Theta_{2}$. 
5.3. The comparison principle for admissible viscosity solutions. We conclude with a few remarks on the structural conditions to impose on $F$ in order to ensure the validity of the comparison principle.

Remark 5.15. As noted in Remark 5.10, the standard structural condition (5.12)-(5.13) may fail in cases where (1.19) holds. On the other hand, (1.19) can fail in cases where (5.12)-(5.13) holds. For example, this happens generically for linear equations

$$
F(x, A)=\operatorname{tr}[a(x) A]-f(x) .
$$

The approach of using elliptic branches following the path initiated by Harvey and Lawson in $[\mathbf{1 1}]$ is truly a nonlinear theory. See also the Cautionary Note 2.7 in Section 2 of their subsequent paper [12]. If $a(x) \geq 0$ is not a constant coefficient matrix, then the natural elliptic map $\Theta$ given by $\Theta(x)=\{A \in \mathcal{S}(N): F(x, A) \geq 0\}$ takes values in a half space with inclination that depends on $a(x)$. As $a(x)$ varies, the hyperplane boundaries of $\Theta(x)$ will be divergent as $\|A\| \rightarrow \infty$ so that the uniform Hausdorff continuity (3.8) will typically fail. On the other hand, (5.12)-(5.13) is satisfied provided that $a=\sigma^{T} \sigma$ with $\sigma$ a Lipschitz matrix. If $\sigma$ fails to be Lipschitz, the comparison principle may fail (see Section 3 of Ishii [14]). In Ishii's counterexample, the two viscosity solutions have unbounded Hessians, which is the reason that $F$ defined by (5.16) will not satisfy our structure condition (1.19).

However, by truncating $F$ where the Hessian could be large, one can force (5.16) into the present theory without assuming that $a=\sigma^{T} \sigma$ with $\sigma$ Lipschitz. For example, assume that

$$
\begin{aligned}
a(x) \in \mathcal{B}_{\lambda, \Lambda}:=\left\{\beta \in \mathcal{S}(N): \lambda \leq \lambda_{1}(\beta) \leq \cdots \leq\right. & \left.\lambda_{N}(\beta) \leq \Lambda\right\} \\
& \text { for each } x \in \Omega
\end{aligned}
$$

with $0<\lambda \leq \Lambda<+\infty$. For $h \in \mathbb{R}$ define the truncation $F_{h}: \Omega \times \mathcal{S}(N) \rightarrow$ $\mathbb{R}$ by

$$
F_{h}(x, A):=\min \left\{F(x, A), \mathcal{M}_{\frac{\lambda}{2}, \Lambda}^{-}(A)+h\right\}
$$

where

$$
\mathcal{M}_{\lambda, \Lambda}^{-}(A):=\inf _{\beta \in \mathcal{B}_{\lambda, \Lambda}} \operatorname{tr}[\beta A]=\lambda \sum_{\lambda_{k}(A)>0} \lambda_{k}(A)-\Lambda \sum_{\lambda_{k}(A)<0} \lambda_{k}(A)
$$

is Pucci's minimal operator.

Remark 5.16. Let $F$ be of the form (5.16) with $f \in \mathrm{UC}(\Omega, \mathbb{R}), a \in$ $\mathrm{UC}(\Omega, \mathcal{S}(N))$ a non constant map satisfying (5.17) and $F_{h}$ defined 
by (5.18). Then one can easily show that the set valued map $\Theta_{h}: \Omega \rightarrow$ $\mathcal{S}(N)$ defined by

$$
\Theta_{h}(x):=\left\{A \in \mathcal{S}(N): F_{h}(x, A) \geq 0\right\}
$$

is a uniformly Hausdorff continuous elliptic map, and hence the comparison principle of Theorem 1.1 holds for $\Theta_{h}$. Moreover, since $F_{h}(x, \cdot)$ is concave and uniformly elliptic, one has Hölder regularity theory for the viscosity solutions of $F_{h}\left(x, D^{2} u\right)=0$ provided that $a \in C^{0, \alpha}(\bar{\Omega})$ with $\alpha \in(0,1)$ (e.g., see Chapter 8 of Caffarelli-Cabré $[\mathbf{5}]$ ). In particular, viscosity solutions are classical solutions with a uniform bound on the Hessian and hence $F_{h}$ reduces to $F$ for each $h$ such that $h \geq \bar{h}(N, \lambda, \Lambda, \alpha)$. In this admittedly unnatural way, one can prove uniqueness for a uniformly elliptic linear equation using the intrinsically nonlinear theory initiated by Harvey and Lawson.

Acknowledgements. The authors are partially supported the Gruppo Nazionale per l'Analisi Matematica, la Probabilità e le loro Applicazioni (GNAMPA) of the Istituto Nazionale di Alta Matematica (INdAM), the Fondazione CaRiPaRo Project "Nonlinear Partial Differential Equations: models, analysis, and control-theoretic problems", and the INdAM Project 2016 "Fenomeni di segregazione in sistemi stazionari di tipo Mean Field Games a piú populazioni" (Cirant) and the INdAM Project 2016 "Analysis and developments for fully nonlinear equations via the Maximum Principle" (Payne). The authors wish to thank the anonymous referees for their constructive criticism of previous versions of this manuscript.

\section{References}

[1] J.-P. Aubin And A. Cellina, "Differential Inclusions. Set-Valued Maps and Viability Theory", Grundlehren der Mathematischen Wissenschaften 264, Springer-Verlag, Berlin, 1984. DOI: 10.1007/ 978-3-642-69512-4.

[2] M. Bardi and P. Mannucci, On the Dirichlet problem for non-totally degenerate fully nonlinear elliptic equations, Commun. Pure Appl. Anal. 5(4) (2006), 709-731. DOI: 10.3934/cpaa.2006.5.709.

[3] G. Barles AND J. Busca, Existence and comparison results for fully nonlinear degenerate elliptic equations without zeroth-order term, Comm. Partial Differential Equations 26(11-12) (2001), 2323-2337. DOI : 10.1081/PDE-100107824. 
[4] D. Burago, Y. Burago, and S. Ivanov, "A Course in Metric Geometry", Graduate Studies in Mathematics 33, American Mathematical Society, Providence, RI, 2001. DOI : 10.1090/gsm/033.

[5] L. A. Caffarelli And X. Cabré, "Fully Nonlinear Elliptic Equations", American Mathematical Society Colloquium Publications 43, American Mathematical Society, Providence, RI, 1995. DOI : $10.1090 / \operatorname{coll} / 043$.

[6] L. Caffarelli, L. Nirenberg, and J. Spruck, The Dirichlet problem for nonlinear second-order elliptic equations I. MongeAmpère equation, Comm. Pure Appl. Math. 37(3) (1984), 369-402. DOI : $10.1002 /$ cpa.3160370306.

[7] L. Caffarelli, L. Nirenberg, and J. Spruck, The Dirichlet problem for nonlinear second-order elliptic equations, III: Functions of the eigenvalues of the Hessian, Acta Math. 155(3-4) (1985), 261-301. DOI: $10.1007 / \mathrm{BF} 02392544$.

[8] M. G. Crandall and H. Ishit, The maximum principle for semicontinuous functions, Differential Integral Equations 3(6) (1990), 1001-1014.

[9] M. G. Crandall, H. Ishit, And P.-L. Lions, User's guide to viscosity solutions of second order partial differential equations, Bull. Amer. Math. Soc. (N.S.) 27(1) (1992), 1-67. DOI: 10.1090/S0273-0979-1992-00266-5.

[10] R. L. Foote, Regularity of the distance function, Proc. Amer. Math. Soc. 92(1) (1984), 153-155. DOI: $10.2307 / 2045171$.

[11] F. R. Harvey and H. B. Lawson, JR., Dirichlet duality and the nonlinear Dirichlet problem, Comm. Pure Appl. Math. 62(3) (2009), 396-443. DOI: 10.1002/cpa. 20265.

[12] F. R. Harvey and H. B. Lawson, JR., Dirichlet duality and the nonlinear Dirichlet problem on Riemannian manifolds, J. Differential Geom. 88(3) (2011), 395-482.

[13] F. R. Harvey and H. B. Lawson, JR., Notes on the differentiation of quasi-convex functions, Preprint (2013). arXiv:1309.1772.

[14] H. IsHit, On uniqueness and existence of viscosity solutions of fully nonlinear second-order elliptic PDEs, Comm. Pure Appl. Math. 42(1) (1989), 15-45. DOI: 10.1002/cpa.3160420103.

[15] H. Ishin AND P.-L. LiOns, Viscosity solutions of fully nonlinear second-order elliptic partial differential equations, J. Differential Equations 83(1) (1990), 26-78. DOI: 10.1016/0022-0396(90)90068 $-\mathrm{Z}$. 
[16] R. Jensen, The maximum principle for viscosity solutions of fully nonlinear second order partial differential equations, Arch. Rational Mech. Anal. 101(1) (1988), 1-27. DOI: 10.1007/BF00281780.

[17] B. Kawohl and N. KuTEv, Comparison principle for viscosity solutions of fully nonlinear, degenerate elliptic equations, Comm. Partial Differential Equations 32(7-9) (2007), 1209-1224. DOI: 10.1080/03605300601113043.

[18] N. V. Krylov, "Nonlinear Elliptic and Parabolic Equations of the Second Order", Translated from the Russian by P. L. Buzytsky [P. L. Buzytski1], Mathematics and its Applications (Soviet Series) 7, D. Reidel Publishing Co., Dordrecht, 1987.

[19] N. V. KRYLov, "Lectures on Fully Nonlinear Second Order Elliptic Equations", Rudolph- Lipschitz-Vorlesung 29, Rheinische FriedrichWilhelms Universität, Bonn, 1994.

[20] N. V. KRYLOV, On the general notion of fully nonlinear secondorder elliptic equations, Trans. Amer. Math. Soc. 347(3) (1995), 857-895. DOI: $10.2307 / 2154876$.

[21] Y. Luo AND A. Eberhard, Comparison principles for viscosity solutions of elliptic equations via fuzzy sum rule, J. Math. Anal. Appl. 307(2) (2005), 736-752. DOI: 10.1016/j.jmaa.2005.01.055.

[22] Z. Slodkowski, The Bremermann-Dirichlet problem for $q$ plurisubharmonic functions, Ann. Scuola Norm. Sup. Pisa Cl. Sci. (4) 11(2) (1984), 303-326.

[23] N. S. TRudinger, The Dirichlet problem for the prescribed curvature equations, Arch. Rational Mech. Anal. 111(2) (1990), 153-179. DOI: $10.1007 / \mathrm{BF} 00375406$.

[24] J. B. WALSh, Continuity of envelopes of plurisubharmonic functions, J. Math. Mech. 18 (1968/1969), 143-148.

Dipartimento di Matematica "F. Enriques"

Università di Milano

Via C. Saldini 50

20133-Milano

Italy

E-mail address: marco.cirant@unimi.it

E-mail address: kevin.payne@unimi.it

Primera versió rebuda el 15 de febrer de 2016, darrera versió rebuda el 27 de maig de 2016. 